Supplementary material for the manuscript

Structural Chemistry \& Number Theory Amalgamized:

The Crystal Structure of $\mathrm{Na}_{11} \mathrm{Hg}_{52}$

authored by

Wolfgang Hornfeck and Constantin Hoch

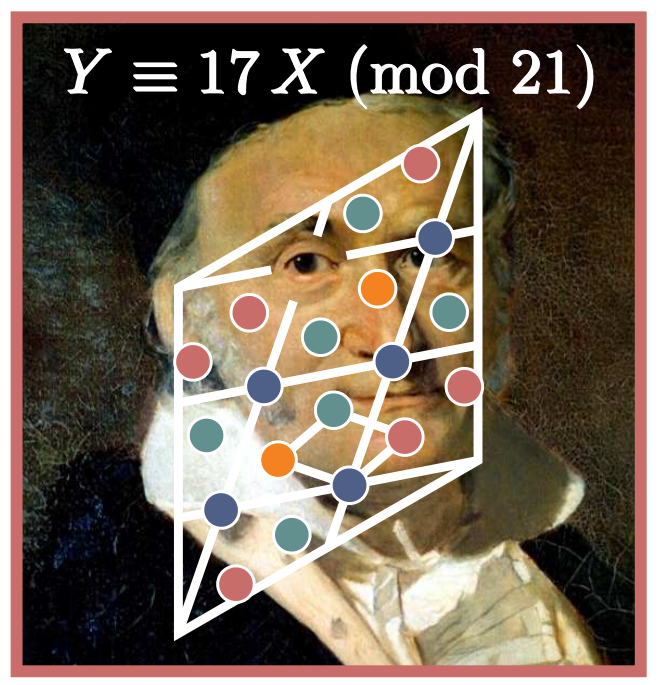

Gaussian spectacles The remarkably complex crystal structure of the sodium amalgam $\mathrm{Na}_{11} \mathrm{Hg}_{52}$ is best described via group-theoretic sublattices which are generated and concisely represented, including their point group symmetry, by number-theoretic formulas taken from the field of modular arithmetic developed by Gauss.

\title{
Contents
}

1 Description of the crystal structure of $\mathrm{Na}_{11} \mathrm{Hg}_{52}$ in terms of multiplicative congruential generators (MCGs) 2

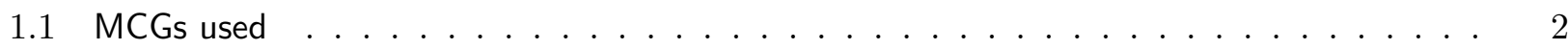

1.1.1 $P / P^{\prime}$-layers, $\mathrm{Na}$ - \& non-rotated $\mathrm{Hg}$-substructure of $F 1 / F 2$-layers $\ldots \ldots \ldots$

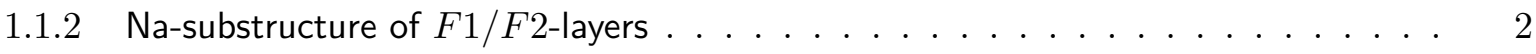

1.1.3 Rotated $\mathrm{Hg}$-substructure of $\mathrm{F} 1 / \mathrm{F}$ 2-layers $\ldots \ldots \ldots \ldots \ldots \ldots$

1.2 Site occupancy schemes of $F 1 / F 2$-layers $\ldots \ldots \ldots \ldots \ldots \ldots$

1.2.1 Site occupancy scheme in 'circular twist' representation . . . . . . . . . . . . . . . . . 4

1.2 .2 Site occupancy scheme in 'linear shift' representation . . . . . . . . . . . . 5

1.2 .3 Group-subgroup scheme . . . . . . . . . . . . . . . . . 6

2 Remarks on the diffraction pattern of $\mathrm{Na}_{11} \mathbf{H g}_{52} \quad \mathbf{7}$

2.1 Selected reciprocal space sections of $\mathrm{Na}_{11} \mathrm{Hg}_{52} \ldots \ldots \ldots \ldots \ldots \ldots$

2.2 Schematic diffraction patterns $h k 0$ of sublattices and partial structures used for the description of $\mathrm{Na}_{11} \mathrm{Hg}_{52} \quad \ldots \ldots \ldots \ldots \ldots \ldots \ldots \ldots \ldots \ldots$

$\begin{array}{lll}3 & \text { Selection of enlarged figures } & 15\end{array}$ 


\section{Description of the crystal structure of $\mathrm{Na}_{11} \mathrm{Hg}_{52}$ in terms of multiplicative congruential generators (MCGs)}

The following sections contain selected information about the MCGs used for the description of the crystal structure of $\mathrm{Na}_{11} \mathrm{Hg}_{52}$. Given are the sublattice index identical to the modulus of the MCG, $T(\alpha, \beta)=M$, followed by its formula $Y=m X(\bmod M)$ with respect to integer coordinates $(X, Y)$. Fractional coordinates of the sublattice nodes are obtained as $(x, y)=(X, Y) / M$. Finally, each MCG is explicitely stated in its canonical ordering of cycles.

\section{$1.1 \quad$ MCGs used}

\subsection{1 $P / P^{\prime}$-layers, Na- \& non-rotated Hg-substructure of $F 1 / F 2$-layers}

$T(14,1)=183 ; Y \equiv 14 X(\bmod 183) ;$

List of generators : $\{1, \cdots, 12,16, \cdots, 24,31, \cdots, 36,46, \cdots, 48,61\}$;

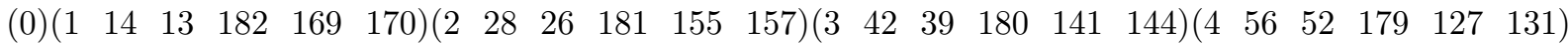

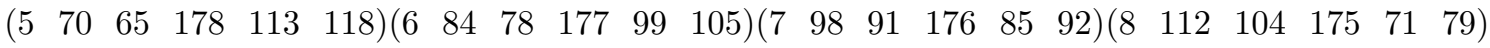

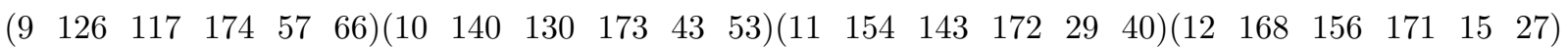

$\left(\begin{array}{lllllllllllllllllllllll}16 & 41 & 25 & 167 & 142 & 158\end{array}\right)\left(\begin{array}{llllllllll}17 & 55 & 38 & 166 & 128 & 145\end{array}\right)\left(\begin{array}{lllllll}18 & 69 & 51 & 165 & 114 & 132\end{array}\right)\left(\begin{array}{llllll}19 & 83 & 64 & 164 & 100 & 119\end{array}\right)$

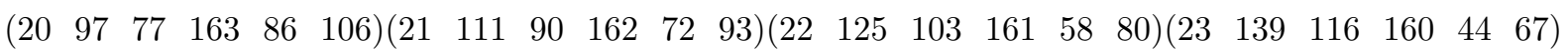

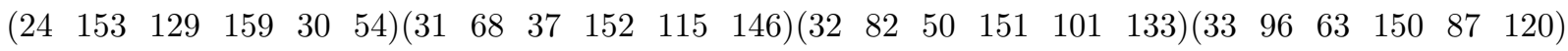

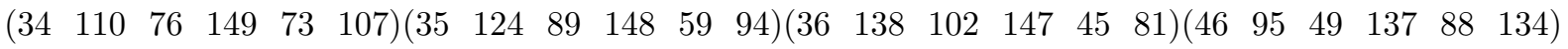

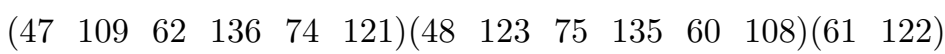

\subsubsection{Na-substructure of $F 1 / F 2$-layers}

$T(9,5)=61 ; Y \equiv 14 X(\bmod 61) ;$

List of generators : $\{1, \cdots, 4,6, \cdots, 8,11 \& 12,16\}$;

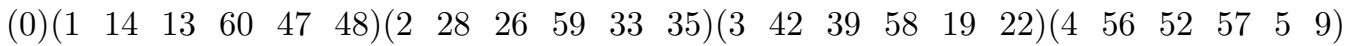

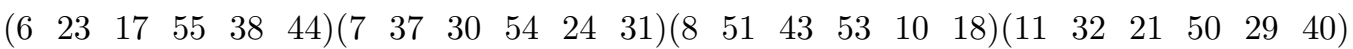

$\left(\begin{array}{llllll}12 & 46 & 34 & 49 & 15 & 27\end{array}\right)\left(\begin{array}{llllll}16 & 41 & 25 & 45 & 20 & 36\end{array}\right)$

\subsubsection{Rotated Hg-substructure of $F 1 / F 2$-layers}

$T(15,7)=169 ; Y \equiv 147 X(\bmod 169) ;$

List of generators : $\{1, \cdots, 7,9, \cdots, 14,17, \cdots, 21,25, \cdots, 28,33, \cdots, 35,41 \& 42,49\}$;

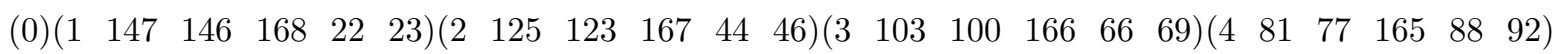

$\left(\begin{array}{llllllllllllllllll}5 & 59 & 54 & 164 & 110 & 115)(6 & 37 & 31 & 163 & 132 & 138)(7 & 15 & 8 & 162 & 154 & 161\end{array}\right)\left(\begin{array}{lllllll}9 & 140 & 131 & 160 & 29 & 38\end{array}\right)$

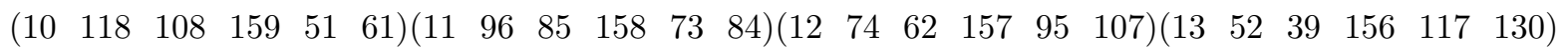

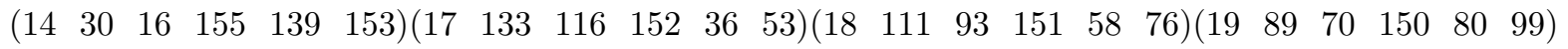

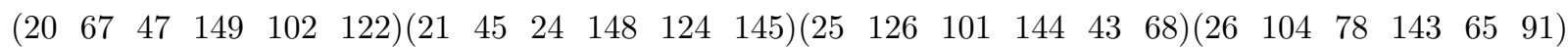

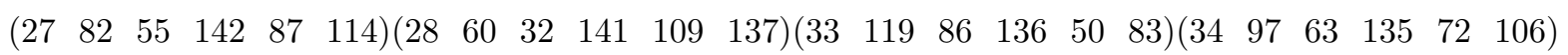

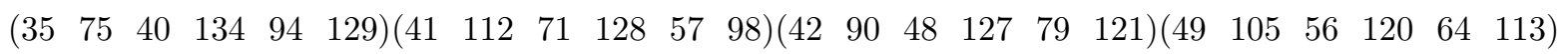




\subsection{Site occupancy schemes of $F 1 / F 2$-layers}

In the following we give some detailed information about the site occupancy scheme as it is occurring in the flat layers, $F 1 / F 2$, of the crystal structure of $\mathrm{Na}_{11} \mathrm{Hg}_{52}$. In particular, we thereby establish a concordance between:

1. The cycle structure of the multiplicative congruential generator $Y \equiv 14 X(\bmod 183)$,

2. its expansion into integral coordinate pairs $\left(\begin{array}{ll}X & Y\end{array}\right)_{F 2}^{F 1}$, and

3. the site-specific chemical decoration of the two distinct flat layers with the elements $\mathrm{Na}(\mathrm{\circ})$ and $\mathrm{Hg}(\bullet)$, including their occurrence on the same site with mixed occupancy $(\circ / \bullet)$ or the presence of vacancies $(\square)$.

As before, fractional coordinates are obtained as: $(x, y)=(X, Y) / M$ where $M=183$.

Moreover, the site occupancy scheme is given in two representations,

1. A 'circular twist' one, in which sites are arranged according to the one-line cycle representation of an MCG, and

2. a 'linear shift' one, in which sites are arranged according to the two-line matrix representation of an MCG.

Both representations highlight distinct features of the structure, regarding the observed distribution of atoms and vacancies. The 'circular twist' representation, for instance, matches the crystallographic orbits, i.e. the splitting of sublattice sites according to space group symmetry. Listing the coordinate pairs $\left(\begin{array}{ll}X & Y\end{array}\right)_{F 2}^{F 1}$ in a 'linear shift' fashion, on the other hand, reveals a general distribution pattern

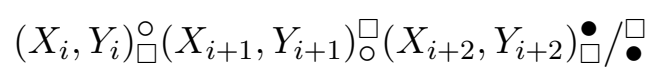

for the site occupancies of successive triples, starting from the origin, which may have been left unnoticed otherwise.

The numerous (colour-coded) exceptions to this general distribution pattern, which are responsible for the lion's share of the structural complexity of $\mathrm{Na}_{11} \mathrm{Hg}_{52}$, are due to vacancy formation $(\circ \rightarrow \square$ or $\bullet \rightarrow \square$ ), while only a few ones are related to a distinct chemical ordering (always $\circ \rightarrow \bullet$, never - $\rightarrow$ o). Yet still 115 out of 183 sites (62.8\%) match the expected atomic species distribution pattern, i.e. $\left(\mathrm{Na}_{2} \mathrm{Hg}\right)_{61}$, for the combined flat layers along a torus line starting from the origin and proceeding along the $[1,14,0]$ direction.

Finally, from yet another point of view, a more conventional group-subgroup scheme covers the splitting of Wyckoff positions and the accompanying hierarchical evolution of the site occupancies as resulting from a sequence of symmetry reduction steps, starting from a hexagonal primitive aristotype structure. Here, the site occupancy scheme observed for $\mathrm{Na}_{11} \mathrm{Hg}_{52}$ turns out to be the outcome of a chemically meaningful ordering along the line

$$
\mathrm{Na} / \mathrm{Hg} \rightarrow \mathrm{NaHg} \rightarrow \mathrm{Na}_{2} \mathrm{Hg} \rightarrow \mathrm{NaHg}_{5} \rightarrow \ldots
$$

up to the introduction of vacancies yielding the actual crystal structure of $\mathrm{Na}_{11} \mathrm{Hg}_{52}$. 


\subsubsection{Site occupancy scheme in 'circular twist' representation}

\begin{tabular}{|c|c|}
\hline$(X \quad Y)_{F 2}^{F 1}$ & 3) \\
\hline$\left(\begin{array}{ll}0 & 0\end{array}\right)_{\square}^{\bullet}$ & $(0)$ \\
\hline$(1 \quad 14)_{\circ}^{\square}(14 \quad 13)_{\square}^{\bullet}(13 \quad 182)_{\bigcirc}^{\square}(182 \quad 169)_{\square}^{\bullet}(169 \quad 170)_{\bigcirc}^{\square}\left(\begin{array}{lll}170 & 1\end{array}\right)_{\square}^{\bullet}$ & $\left(\begin{array}{llllll}1 & 14 & 13 & 182 & 169 & 170\end{array}\right)$ \\
\hline$(2 \quad 28) \square(28 \quad 26) \square(26 \quad 181) \square(181 \quad 155)_{\square}^{\square}(155 \quad 157)_{\square}^{\square}\left(\begin{array}{lll}157 & 2\end{array}\right)_{\bigcirc}^{\square}$ & $\left(\begin{array}{llllll}2 & 28 & 26 & 181 & 155 & 157\end{array}\right)$ \\
\hline$(3 \quad 42)_{\square}^{\square}(42 \quad 39)_{\square}^{\circ}(39 \quad 180)_{\square}^{\square}(180 \quad 141)_{\square}^{\circ}(141 \quad 144)_{\square}^{\square}(144 \quad 3)_{\square}^{\circ}$ & $\left(\begin{array}{llllll}3 & 42 & 39 & 180 & 141 & 144\end{array}\right)$ \\
\hline$(4 \quad 56)_{\triangleright}^{\square}(56 \quad 52)_{\square}^{\bullet}(52 \quad 179)_{\bigcirc}^{\square}(179 \quad 127)_{\square}^{\bullet}(127 \quad 131)^{\square}(131 \quad 4)_{\square}^{\bullet}$ & $\left(\begin{array}{llllll}4 & 56 & 52 & 179 & 127 & 131\end{array}\right)$ \\
\hline 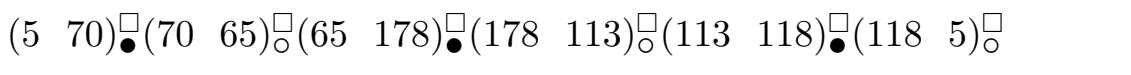 & $\left(\begin{array}{llllll}5 & 70 & 65 & 178 & 113 & 118\end{array}\right)$ \\
\hline$(6 \quad 84)_{\square}^{\circ}(84 \quad 78)_{\square}^{\square}(78 \quad 177)_{\square}^{\circ}(177 \quad 99)_{\square}^{\square}(99 \quad 105)_{\square}^{\circ}(105 \quad 6)_{\square}^{\square}$ & $\left(\begin{array}{llllll}6 & 84 & 78 & 177 & 99 & 105\end{array}\right)$ \\
\hline$(7 \quad 98))_{\circ}^{\square}(98 \quad 91) \square(91 \quad 176)_{\square}^{\square}(176 \quad 85)_{\square}^{\square}(85 \quad 92)_{\circ}^{\square}(92 \quad 7)_{\square}^{\square}$ & $\left(\begin{array}{llllll}7 & 98 & 91 & 176 & 85 & 92\end{array}\right)$ \\
\hline$(8 \quad 112)_{\bullet}^{\square}(112 \quad 104)_{\circ}^{\square}(104 \quad 175)^{\square}(175 \quad 71)_{\circ}^{\square}(71 \quad 79)_{\bullet}^{\square}(79 \quad 8)_{\circ}^{\square}$ & $\left(\begin{array}{llllll}8 & 112 & 104 & 175 & 71 & 79\end{array}\right)$ \\
\hline$(9 \quad 126)_{\square}^{\circ}(126 \quad 117)_{\square}^{\circ}(117 \quad 174)_{\square}^{\circ}(174 \quad 57)_{\square}^{\circ}(57 \quad 66)_{\square}^{\circ}(66 \quad 9)_{\square}^{\circ}$ & $\left(\begin{array}{llllll}9 & 126 & 117 & 174 & 57 & 66\end{array}\right)$ \\
\hline 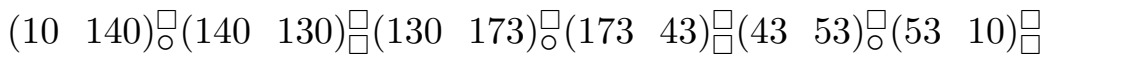 & $\left(\begin{array}{llllll}10 & 140 & 130 & 173 & 43 & 53\end{array}\right)$ \\
\hline$(11 \quad 154) \bullet(154 \quad 143) \square(143 \quad 172) \bullet(172 \quad 29) \square(29 \quad 40) \square(40 \quad 11) \square$ & $\left(\begin{array}{llllll}11 & 154 & 143 & 172 & 29 & 40\end{array}\right)$ \\
\hline$(12 \quad 168)_{\square}^{\circ}(168 \quad 156)_{\square}^{\square}(156 \quad 171)_{\square}^{\circ}(171 \quad 15)_{\square}^{\natural}(15 \quad 27)_{\square}^{\circ}(27 \quad 12)_{\square}^{\square}$ & $\left(\begin{array}{llllll}12 & 168 & 156 & 171 & 15 & 27\end{array}\right)$ \\
\hline$(16 \quad 41)^{\square}(41 \quad 25)_{\square}^{\square}(25 \quad 167)_{\bigcirc}^{\square}\left(\begin{array}{lll}167 & 142\end{array}\right)_{\square}^{\square}(142 \quad 158)^{\square}(158 \quad 16)_{\square}^{\square}$ & $\left(\begin{array}{llllll}16 & 41 & 25 & 167 & 142 & 158\end{array}\right)$ \\
\hline$(17 \quad 55)_{\square}^{\bullet}(55 \quad 38)_{\bigcirc}^{\square}(38 \quad 166)_{\square}^{\bullet}(166 \quad 128)_{\bigcirc}^{\square}(128 \quad 145)_{\square}^{\bullet}(145 \quad 17)_{\circ}^{\square}$ & $\left(\begin{array}{llllll}17 & 55 & 38 & 166 & 128 & 145\end{array}\right)$ \\
\hline$(18 \quad 69)_{\square}^{\circ}(69 \quad 51)_{\square}^{\circ}(51 \quad 165)_{\square}^{\circ}(165 \quad 114)_{\square}^{\circ}(114 \quad 132)_{\square}^{\circ}(132 \quad 18)_{\square}^{\circ}$ & $\left(\begin{array}{llllll}18 & 69 & 51 & 165 & 114 & 132\end{array}\right)$ \\
\hline$(19 \quad 83)_{\circ}^{\square}(83 \quad 64)_{\square}^{\square}(64 \quad 164)_{\circ}^{\square}(164 \quad 100) \square(100 \quad 119)_{\bigcirc}^{\square}(119 \quad 19)_{\square}^{\square}$ & $\left(\begin{array}{llllll}19 & 83 & 64 & 164 & 100 & 119\end{array}\right)$ \\
\hline$(20 \quad 97)_{\square}^{\bullet}(97 \quad 77)_{\square}^{\square}(77 \quad 163)_{\square}^{\bullet}(163 \quad 86)_{\square}^{\square}(86 \quad 106)_{\square}^{\bullet}(106 \quad 20)$ 吕 & $\left(\begin{array}{llllll}20 & 97 & 77 & 163 & 86 & 106\end{array}\right)$ \\
\hline$(21 \quad \text { 111) })_{\square}^{\circ}(111 \quad 90) \square(90 \quad 162)_{\square}^{\circ}\left(\begin{array}{ll}162 & 72\end{array}\right)_{\square}^{\square}(72 \quad 93)_{\square}^{\circ}(93 \quad 21)_{\square}^{\square}$ & $\left(\begin{array}{llllll}21 & 111 & 90 & 162 & 72 & 93\end{array}\right)$ \\
\hline$(22 \quad 125)_{\square}^{\square}(125 \quad 103)_{\square}^{\bullet}(103 \quad 161)_{\square}^{\square}(161 \quad 58)_{\square}^{\bullet}(58 \quad 80)_{\square}^{\square}(80 \quad 22)_{\square}^{\bullet}$ & $\left(\begin{array}{llllll}22 & 125 & 103 & 161 & 58 & 80\end{array}\right)$ \\
\hline$(23 \quad 139)_{\square}^{\square}(139 \quad 116)_{\circ}^{\square}(116 \quad 160)_{\square}^{\square}(160 \quad 44)_{\circ}^{\square}(44 \quad 67)_{\square}^{\square}(67 \quad 23)_{\circ}^{\square}$ & $\left(\begin{array}{llllll}23 & 139 & 116 & 160 & 44 & 67\end{array}\right)$ \\
\hline$(24 \quad 153)_{\square}^{\circ}(153 \quad 129)_{\square}^{\square}(129 \quad 159)_{\square}^{\circ}(159 \quad 30)_{\square}^{\square}(30 \quad 54)_{\square}^{\circ}(54 \quad 24)_{\square}^{\square}$ & $\left(\begin{array}{llllll}24 & 153 & 129 & 159 & 30 & 54\end{array}\right)$ \\
\hline$(31 \quad 68)_{\square}^{\square}(68 \quad 37) \bullet(37 \quad 152) \square(152 \quad 115)_{\bullet}^{\square}(115 \quad 146)_{\square}^{\square}(146 \quad 31)^{\square}$ & $\left(\begin{array}{llllll}31 & 68 & 37 & 152 & 115 & 146\end{array}\right)$ \\
\hline$(32 \quad 82)_{\square}^{\square}(82 \quad 50)_{\bigcirc}^{\square}(50 \quad 151)_{\square}^{\square}\left(\begin{array}{ll}151 & 101\end{array}\right)_{\circ}^{\square}(101 \quad 133)_{\square}^{\square}\left(\begin{array}{ll}133 & 32\end{array}\right)_{\circ}^{\square}$ & $\left(\begin{array}{llllll}32 & 82 & 50 & 151 & 101 & 133\end{array}\right)$ \\
\hline$(33 \quad 96)_{\square}^{\circ}(96 \quad 63)_{\square}^{\square}(63 \quad 150)_{\square}^{\circ}(150 \quad 87)_{\square}^{\square}(87 \quad 120)_{\square}^{\circ}(120 \quad 33)_{\square}^{\square}$ & $\left(\begin{array}{llllll}33 & 96 & 63 & 150 & 87 & 120\end{array}\right)$ \\
\hline$(34 \quad 110)_{\bigcirc}^{\square}(110 \quad 76)_{\square}^{\square}(76 \quad 149)_{\bigcirc}^{\square}(149 \quad 73)_{\square}^{\square}(73 \quad 107)_{\bigcirc}^{\square}(107 \quad 34)_{\square}^{\square}$ & $\left(\begin{array}{llllll}34 & 110 & 76 & 149 & 73 & 107\end{array}\right)$ \\
\hline 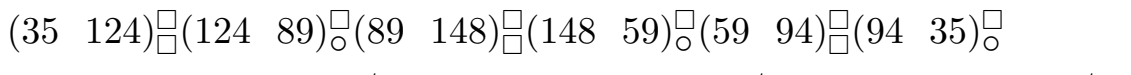 & $\left(\begin{array}{llllll}35 & 124 & 89 & 148 & 59 & 94\end{array}\right)$ \\
\hline$(36 \quad 138)_{\square}^{\square}(138 \quad 102)_{\square}^{\circ / \bullet}(102 \quad 147)_{\square}^{\square}(147 \quad 45)_{\square}^{\circ / \bullet}(45 \quad 81)_{\square}^{\square}(81 \quad 36)_{\square}^{\circ / \bullet}$ & $\left(\begin{array}{llllll}36 & 138 & 102 & 147 & 45 & 81\end{array}\right)$ \\
\hline (46 95$)_{\square}^{\square}(95 \quad 49)_{\square}^{\bullet}(49 \quad 137)_{\square}^{\square}(137 \quad 88)_{\square}^{\bullet}(88 \quad 134)_{\square}^{\square}(134 \quad 46)_{\square}^{\bullet}$ & $\left(\begin{array}{llllll}46 & 95 & 49 & 137 & 88 & 134\end{array}\right)$ \\
\hline$(47 \quad 109)_{\bullet}^{\square}(109 \quad 62)_{\bigcirc}^{\square}(62 \quad 136)_{\bullet}^{\square}(136 \quad 74)_{\circ}^{\square}(74 \quad 121)_{\bullet}^{\square}(121 \quad 47)_{\circ}^{\square}$ & $\left(\begin{array}{llllll}47 & 109 & 62 & 136 & 74 & 121\end{array}\right)$ \\
\hline$(48 \quad 123)_{\square}^{\circ}(123 \quad 75)_{\square}^{\circ}(75 \quad 135)_{\square}^{\circ}(135 \quad 60)_{\square}^{\circ}(60 \quad 108)_{\square}^{\circ}(108 \quad 48)_{\square}^{\circ}$ & $\left(\begin{array}{llllll}48 & 123 & 75 & 135 & 60 & 108\end{array}\right)$ \\
\hline$(61 \quad 122) \bullet(122 \quad 61)_{\bullet}^{\bullet}$ & $\left(\begin{array}{ll}61 & 122\end{array}\right)$ \\
\hline
\end{tabular}




\begin{tabular}{|c|c|c|}
\hline & $\left(\begin{array}{ll}X & Y\end{array}\right)_{F 2}^{F 1}$ & \\
\hline$\left(\begin{array}{ll}0 & 0\end{array}\right)_{\square}^{\bullet}$ & $\left(\begin{array}{ll}1 & 14\end{array}\right)_{\bigcirc}^{\square}$ & $\left(\begin{array}{ll}2 & 28\end{array}\right) \square$ \\
\hline$(3 \quad 42)_{\square}^{\square}$ & $(4 \quad 56)_{\bigcirc}^{\square}$ & $\left(\begin{array}{ll}5 & 70\end{array}\right)$ \\
\hline$\left(\begin{array}{ll}6 & 84\end{array}\right)_{\square}^{\circ}$ & $(7 \quad 98)_{\circ}^{\square}$ & $\left(\begin{array}{ll}8 & 112\end{array}\right)_{\bullet}^{\bullet}$ \\
\hline$\left(\begin{array}{ll}9 & 126\end{array}\right)_{\square}^{\circ}$ & $(10 \quad 140)_{\bigcirc}^{\square}$ & $\left(\begin{array}{ll}11 & 154\end{array}\right)_{\bullet}^{\square}$ \\
\hline$(12 \quad 168)_{\square}^{\circ}$ & $(13 \quad 182)_{\bigcirc}^{\square}$ & $\left(\begin{array}{ll}14 & 13\end{array}\right)_{\square}^{\bullet}$ \\
\hline$\left(\begin{array}{ll}15 & 27\end{array}\right)_{\square}^{\circ}$ & $(16 \quad 41)_{\bigcirc}^{\square}$ & $(17 \quad 55)_{\square}^{\bullet}$ \\
\hline$\left(\begin{array}{ll}18 & 69\end{array}\right)_{\square}^{\circ}$ & $(19 \quad 83)_{\circ}^{\square}$ & $(20 \quad 97)_{\square}^{\bullet}$ \\
\hline$\left(\begin{array}{ll}21 & 111\end{array}\right)_{\square}^{\circ}$ & $\left(\begin{array}{ll}22 & 125\end{array}\right)_{\square}^{\square}$ & (23 $\quad 139)^{\square} \square$ \\
\hline$(24 \quad 153)_{\square}^{\circ}$ & $\left(\begin{array}{ll}25 & 167\end{array}\right)_{\bigcirc}^{\square}$ & (26 181$)^{\square}$ \\
\hline$\left(\begin{array}{ll}27 & 12\end{array}\right)_{\square}^{\square}$ & $\left(\begin{array}{ll}28 & 26\end{array}\right)_{\bigcirc}^{\square}$ & $(29 \quad 40)_{\bullet}^{\bullet}$ \\
\hline$(30 \quad 54)_{\square}^{\circ}$ & $(31 \quad 68) \square$ & $\left(\begin{array}{ll}32 & 82\end{array}\right) \square$ \\
\hline$(33 \quad 96)_{\square}^{\circ}$ & $(34 \quad 110)_{\circ}^{\square}$ & $\left(\begin{array}{ll}35 & 124\end{array}\right)_{\square}^{\square}$ \\
\hline$\left(\begin{array}{ll}36 & 138\end{array}\right)_{\square}^{口}$ & $\left(\begin{array}{ll}37 & 152\end{array}\right)_{\square}^{口}$ & $(38 \quad 166)_{\square}^{\bullet}$ \\
\hline$(39 \quad 180)^{\square}$ & $\left(\begin{array}{ll}40 \quad 11\end{array}\right)_{\square}^{\square}$ & $\left(\begin{array}{ll}41 & 25\end{array}\right)$ \\
\hline$(42 \quad 39)_{\square}^{\circ}$ & $(43 \quad 53)_{\bigcirc}^{\square}$ & $\left(\begin{array}{ll}44 & 67\end{array}\right)_{\square}^{\square}$ \\
\hline$(45 \quad 81) \square$ & $(46 \quad 95)_{\square}^{\square}$ & $\left(\begin{array}{ll}47 & 109\end{array}\right)_{\bullet}^{\square}$ \\
\hline$\left(\begin{array}{ll}48 & 123\end{array}\right)_{\square}^{\circ}$ & $\left(\begin{array}{ll}49 & 137\end{array}\right)_{\square}^{\square}$ & $(50 \quad 151)^{\square}$ \\
\hline$\left(\begin{array}{ll}51 & 165\end{array}\right)_{\square}^{\circ}$ & $\left(\begin{array}{ll}52 & 179\end{array}\right)_{\bigcirc}^{\square}$ & $(53 \quad 10) \square$ \\
\hline$(54 \quad 24)_{\square}^{\square}$ & $(55 \quad 38)^{\square}$ & $\left(\begin{array}{ll}56 & 52\end{array}\right)_{\square}^{\bullet}$ \\
\hline$(57 \quad 66)_{\square}^{\circ}$ & $(58 \quad 80) \square$ & (59 94$)_{\square}^{\square}$ \\
\hline$\left(\begin{array}{ll}60 & 108\end{array}\right)_{\square}^{\circ}$ & $\left(\begin{array}{ll}61 & 122\end{array}\right)_{\bullet}^{\natural}$ & $\left(\begin{array}{ll}62 & 136\end{array}\right)_{\bullet}^{\square}$ \\
\hline$\left(\begin{array}{ll}63 & 150\end{array}\right)_{\square}^{\circ}$ & $\left(\begin{array}{ll}64 & 164\end{array}\right)_{\circ}^{\square}$ & $\left(\begin{array}{ll}65 & 178\end{array}\right)_{\bullet}^{\square}$ \\
\hline$\left(\begin{array}{ll}66 & 9\end{array}\right)_{\square}^{\circ}$ & $\left(\begin{array}{ll}67 & 23\end{array}\right)_{\circ}^{\square}$ & $\left(\begin{array}{ll}68 & 37\end{array}\right)^{\bullet}$ \\
\hline$\left(\begin{array}{ll}69 & 51\end{array}\right)_{\square}^{\circ}$ & $\left(\begin{array}{ll}70 & 65\end{array}\right)^{\square}$ & $\left(\begin{array}{ll}71 & 79\end{array}\right)_{\bullet}^{\bullet}$ \\
\hline$\left(\begin{array}{ll}72 & 93\end{array}\right)_{\square}^{\circ}$ & $\left(\begin{array}{ll}73 & 107\end{array}\right)_{\triangleright}^{\square}$ & $\left(\begin{array}{ll}74 & 121\end{array}\right)_{\bullet}^{\square}$ \\
\hline$\left(\begin{array}{ll}75 & 135\end{array}\right)_{\square}^{\circ}$ & $\left(\begin{array}{ll}76 & 149\end{array}\right)_{\bigcirc}^{\square}$ & $\left(\begin{array}{ll}77 & 163\end{array}\right)_{\square}^{\bullet}$ \\
\hline$\left(\begin{array}{ll}78 & 177\end{array}\right)_{\square}^{\circ}$ & $(79 \quad 8)^{\square}$ & $(80 \quad 22)_{\square}^{\bullet}$ \\
\hline$(8136)_{\square}^{\circ / \bullet}$ & $(82 \quad 50)_{\circ}^{\square}$ & $(83 \quad 64)_{\square}^{\square}$ \\
\hline$(84 \quad 78)_{\square}^{\square}$ & $(85 \quad 92)_{\circ}^{\square}$ & $\left(\begin{array}{ll}86 & 106\end{array}\right)_{\square}^{\bullet}$ \\
\hline$\left(\begin{array}{ll}87 & 120\end{array}\right)_{\square}^{\circ}$ & $(88 \quad 134)_{\square}^{\square}$ & (89 148$)^{\square} \square$ \\
\hline$\left(\begin{array}{ll}90 & 162\end{array}\right)_{\square}^{\circ}$ & $\left(\begin{array}{ll}91 & 176\end{array}\right)_{\circ}^{\square}$ & $\left(\begin{array}{ll}92 & 7\end{array}\right)_{\square}^{\square}$ \\
\hline
\end{tabular}

\begin{tabular}{|c|c|c|}
\hline & $\begin{array}{l}X \quad Y)_{F 2}^{F 1} \\
\end{array}$ & \\
\hline$\left(\begin{array}{ll}93 & 21\end{array}\right)_{\square}^{\square}$ & $(94 \quad 35)^{\square}$ & $(95 \quad 49)_{\square}^{\bullet}$ \\
\hline$(96 \quad 63)_{\square}^{\square}$ & $\left(\begin{array}{ll}97 & 77\end{array}\right)_{\square}^{\square}$ & $\left(\begin{array}{ll}98 & 91\end{array}\right) \square$ \\
\hline$(99 \quad 105)_{\square}^{\circ}$ & $\left(\begin{array}{ll}100 & 119\end{array}\right)_{\bigcirc}^{\square}$ & $\left(\begin{array}{ll}101 \quad 133\end{array}\right)_{\square}^{\square}$ \\
\hline$\left(\begin{array}{ll}102 & 147\end{array}\right)_{\square}^{\square}$ & (103 161 $)_{\square}^{\square}$ & $\left(\begin{array}{ll}104 & 175\end{array}\right)_{\bullet}^{\square}$ \\
\hline$(105 \quad 6)_{\square}^{\square}$ & $(106 \quad 20)_{\square}^{\square}$ & $(107 \quad 34) \square$ \\
\hline$(108 \quad 48)_{\square}^{\circ}$ & $(109 \quad 62)_{\circ}^{\square}$ & $(110 \quad 76)^{\square}$ \\
\hline$(11190)_{\square}^{\square}$ & $\left(\begin{array}{ll}112 & 104\end{array}\right)_{\bigcirc}^{\square}$ & $\left(\begin{array}{ll}113 & 118\end{array}\right)_{\bullet}^{\bullet}$ \\
\hline$\left(\begin{array}{ll}114 & 132\end{array}\right)_{\square}^{\circ}$ & $\left(\begin{array}{ll}115 & 146\end{array}\right)_{\square}^{\square}$ & $\left(\begin{array}{ll}116 \quad 160\end{array}\right)_{\square}^{\square}$ \\
\hline$\left(\begin{array}{ll}117 & 174\end{array}\right)_{\square}^{\circ}$ & $(118 \quad 5)_{\circ}^{\square}$ & $\left(\begin{array}{ll}119 & 19\end{array}\right) \square$ \\
\hline$(120 \quad 33)_{\square}^{\square}$ & $(121 \quad 47)^{\square}$ & $(122 \quad 61)^{\square}$ \\
\hline$(123 \quad 75)_{\square}^{\circ}$ & $(124 \quad 89)_{\bigcirc}^{\square}$ & $(125 \quad 103)_{\square}^{\bullet}$ \\
\hline$(126 \quad 117)_{\square}^{\circ}$ & $\left(\begin{array}{ll}127 & 131\end{array}\right)_{\bigcirc}^{\square}$ & $(128 \quad 145)_{\square}^{\bullet}$ \\
\hline (129 159) & $\left(\begin{array}{ll}130 & 173\end{array}\right)_{\bigcirc}^{\square}$ & $(131 \quad 4)_{\square}^{\bullet}$ \\
\hline$\left(\begin{array}{ll}132 & 18\end{array}\right)_{\square}^{\circ}$ & $(133 \quad 32)_{\circ}^{\square}$ & $(134 \quad 46)_{\square}^{\bullet}$ \\
\hline$(135 \quad 60)_{\square}^{\circ}$ & $(136 \quad 74)_{\bigcirc}^{\square}$ & $(137 \quad 88)_{\square}^{\bullet}$ \\
\hline$\left(\begin{array}{ll}138 & 102\end{array}\right)_{\square}^{\circ / \bullet}$ & $\left(\begin{array}{ll}139 & 116\end{array}\right)_{\circ}^{\square}$ & $\left(\begin{array}{ll}140 & 130\end{array}\right)$ \\
\hline$\left(\begin{array}{ll}141 \quad 144\end{array}\right)_{\square}^{\square}$ & $\left(\begin{array}{ll}142 & 158\end{array}\right)_{\bigcirc}^{\square}$ & $\left(\begin{array}{ll}143 & 172\end{array}\right)_{\bullet}^{\natural}$ \\
\hline$(144 \quad 3)_{\square}^{\circ}$ & $(145 \quad 17)_{\bigcirc}^{\square}$ & $\left(\begin{array}{ll}146 & 31\end{array}\right)_{\bullet}^{\square}$ \\
\hline$(147 \quad 45)_{\square}^{\circ / \bullet}$ & $(148 \quad 59)_{\bigcirc}^{\square}$ & $\left(\begin{array}{ll}149 \quad 73\end{array}\right)_{\square}^{\square}$ \\
\hline$\left(\begin{array}{ll}150 & 87\end{array}\right)_{\square}^{口}$ & $\left(\begin{array}{ll}151 & 101\end{array}\right)_{\bigcirc}^{\square}$ & $\left(\begin{array}{ll}152 & 115\end{array}\right)_{\bullet}^{\natural}$ \\
\hline$\left(\begin{array}{ll}153 & 129\end{array}\right)_{\square}^{\square}$ & $\left(\begin{array}{ll}154 & 143\end{array}\right)_{\square}^{\square}$ & $\left(\begin{array}{ll}155 \quad 157\end{array}\right)$ \\
\hline$(156 \quad 171)_{\square}^{\circ}$ & $\left(\begin{array}{ll}157 & 2\end{array}\right)_{\bigcirc}^{\square}$ & $\left(\begin{array}{ll}158 & 16\end{array}\right)_{\square}^{\square}$ \\
\hline$(159 \quad 30)_{\square}^{\square}$ & $(160 \quad 44)_{\bigcirc}^{\square}$ & $(161 \quad 58)_{\square}^{\bullet}$ \\
\hline$\left(\begin{array}{ll}162 & 72\end{array}\right)_{\square}^{\square}$ & $(163 \quad 86)_{\square}^{\square}$ & $\left(\begin{array}{ll}164 & 100\end{array}\right)_{\square}^{\square}$ \\
\hline$(165 \quad 114)_{\square}^{\circ}$ & $\left(\begin{array}{ll}166 & 128\end{array}\right)_{\circ}^{\square}$ & $\left(\begin{array}{ll}167 & 142\end{array}\right)_{\square}^{\square}$ \\
\hline$(168 \quad 156)_{\square}^{\square}$ & $\left(\begin{array}{ll}169 & 170\end{array}\right)_{\bigcirc}^{\square}$ & $\left(\begin{array}{ll}170 \quad 1\end{array}\right)_{\square}^{\bullet}$ \\
\hline$\left(\begin{array}{ll}171 & 15\end{array}\right)_{\square}^{\square}$ & $\left(\begin{array}{ll}172 \quad 29\end{array}\right)_{\square}^{\square}$ & $(173 \quad 43)^{\square} \square$ \\
\hline$(174 \quad 57)_{\square}^{\circ}$ & $(175 \quad 71)_{\bigcirc}^{\square}$ & $\left(\begin{array}{ll}176 \quad 85\end{array}\right)_{\square}^{\square}$ \\
\hline$\left(\begin{array}{ll}177 & 99\end{array}\right)_{\square}^{\square}$ & $\left(\begin{array}{ll}178 & 113\end{array}\right)_{\circ}^{\square}$ & $\left(\begin{array}{ll}179 \quad 127\end{array}\right)_{\square}^{\bullet}$ \\
\hline$(180 \quad 141)_{\square}^{\circ}$ & $\left(\begin{array}{ll}181 & 155\end{array}\right)_{\circ}^{\square}$ & $\left(\begin{array}{ll}182 \quad 169\end{array}\right)_{\square}^{\bullet}$ \\
\hline
\end{tabular}




\subsubsection{Group-subgroup scheme}

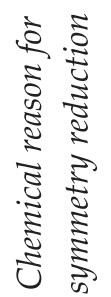
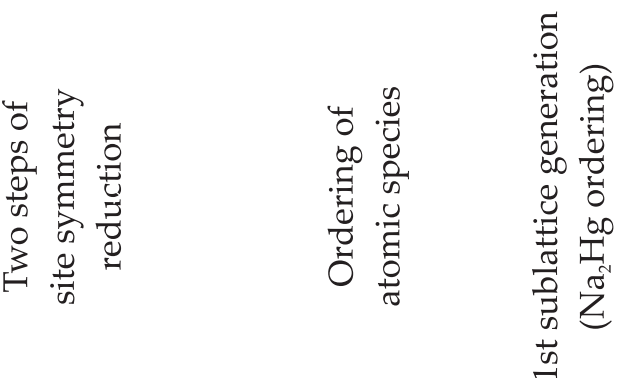

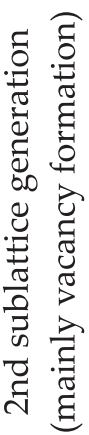

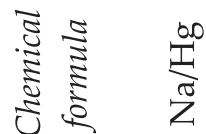

$\stackrel{\infty}{\pi}_{Z}^{\infty} \quad \stackrel{\mathbb{Z}^{\infty}}{\pi}$

$\begin{array}{ll}\prod_{\pi}^{\infty} & \stackrel{0.0}{T^{\pi}} \\ Z & \quad \text { Z }\end{array}$

Z

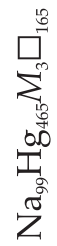

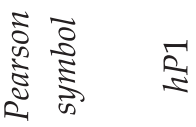

$\Sigma$

$\xi$

$\stackrel{N}{\Xi}$

$\stackrel{0}{\xi}$

辛

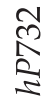

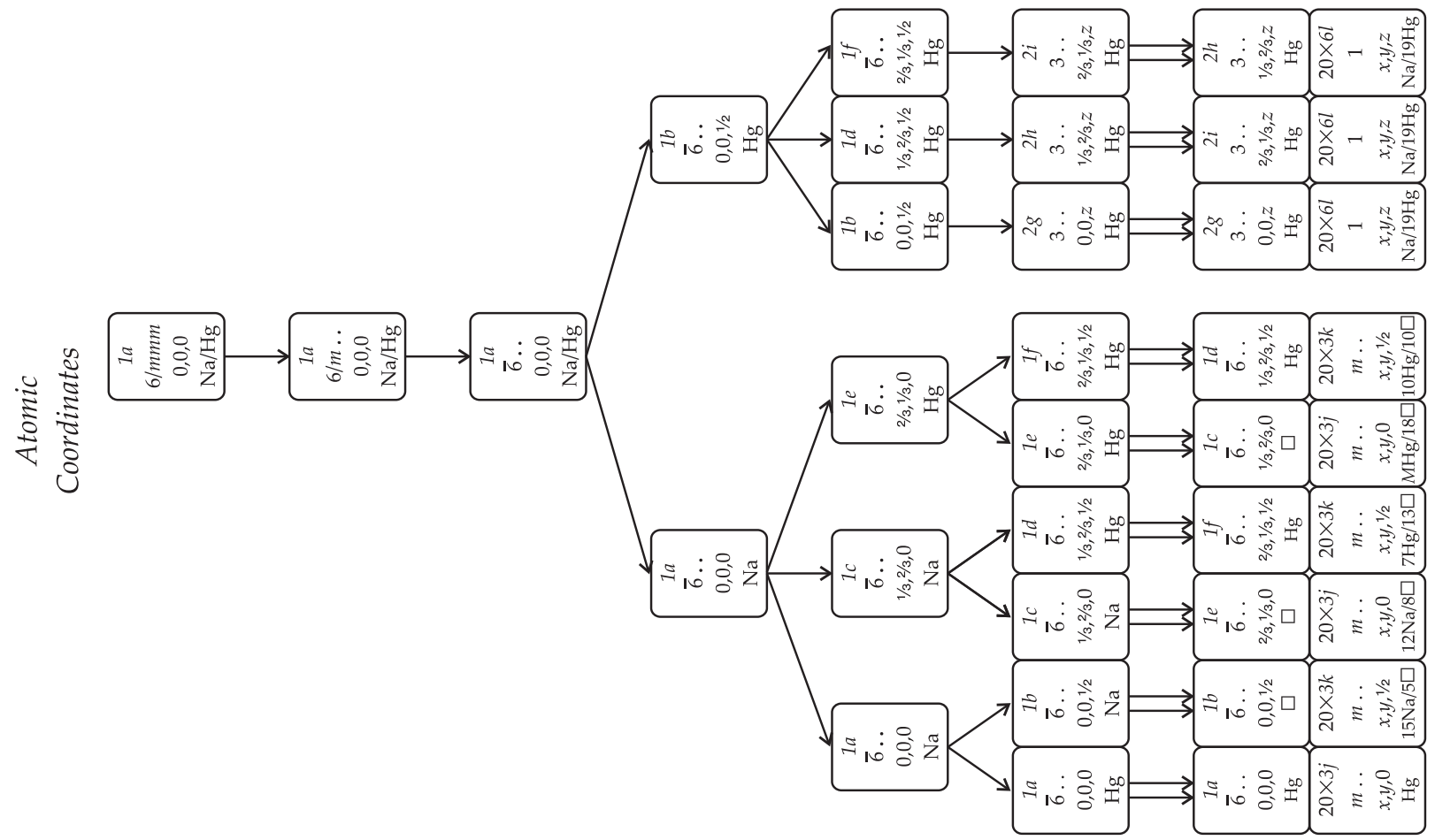

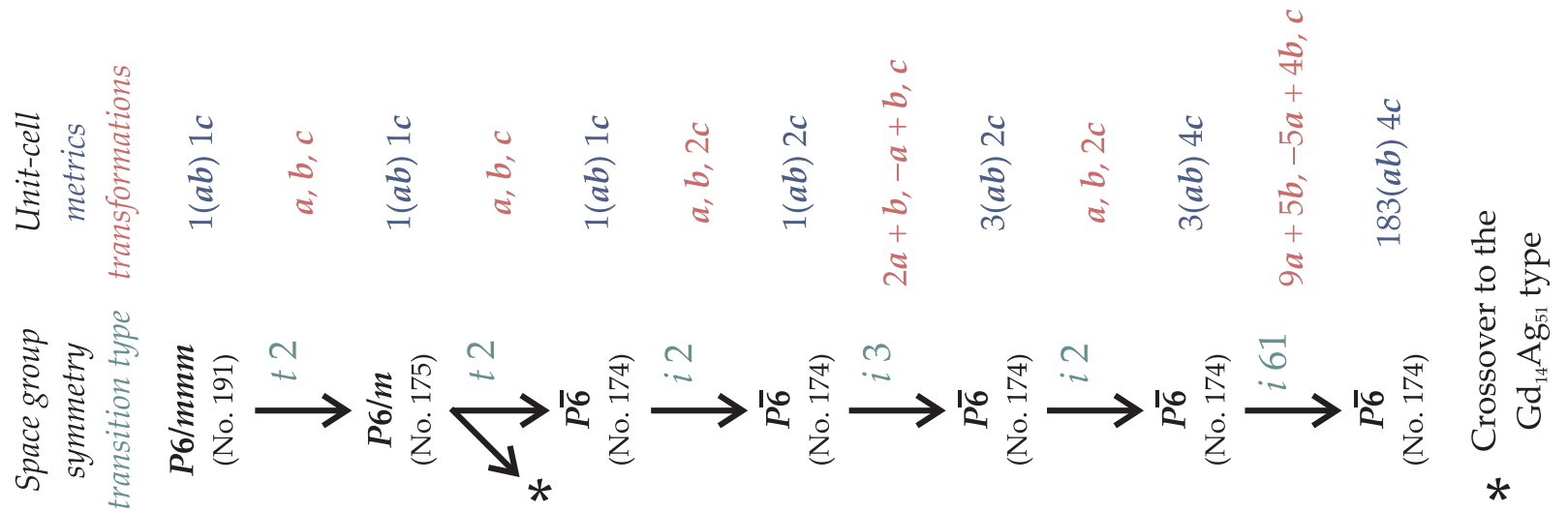




\section{Remarks on the diffraction pattern of $\mathrm{Na}_{11} \mathrm{Hg}_{52}$}

\subsection{Selected reciprocal space sections of $\mathrm{Na}_{11} \mathrm{Hg}_{52}$}

Here we show reconstructed sections of reciprocal space from the actual diffraction experiment performed on $\mathrm{Na}_{11} \mathrm{Hg}_{52}$, which formed the basis for the single-crystal structure solution and refinement of its crystal structure. The keen crystallographer will possibly find quite a few telltale signs of peculiar diffraction phenomena, of which reflections located at wavy 'lattice lines' appear to be the most obvious (see $h 2 l$ in particular), thereby hinting at some possible description as an incommensurately modulated phase. However, the real intention of showing these reciprocal sections is an aesthetical rather than a scientific one: Have fun, while pondering about them!

Figure 1: Reciprocal space sections of $\mathrm{Na}_{11} \mathrm{Hg}_{52}: h k 0$.

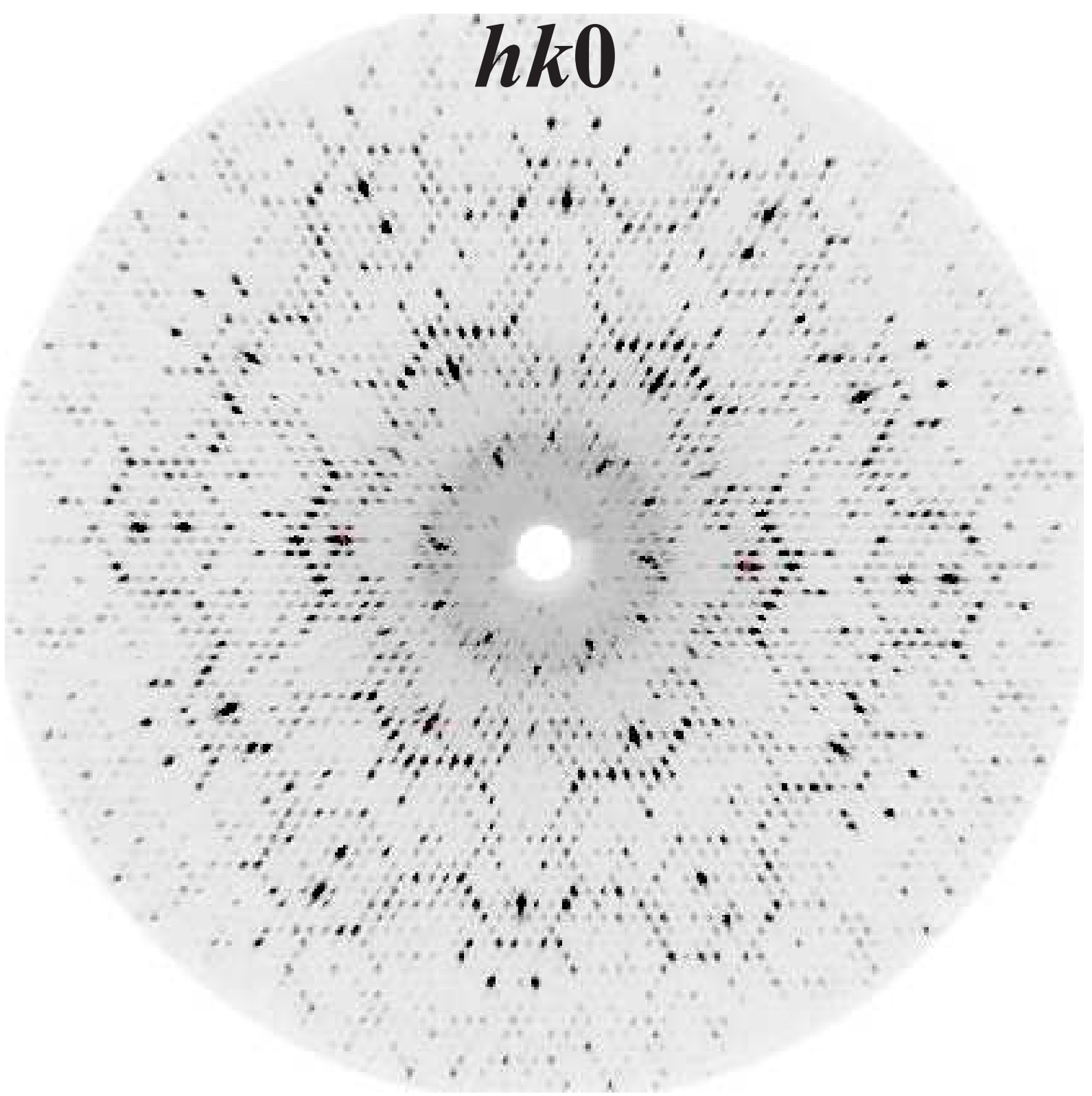


Figure 2: Reciprocal space sections of $\mathrm{Na}_{11} \mathrm{Hg}_{52}: h k l$ to $h k 6$.
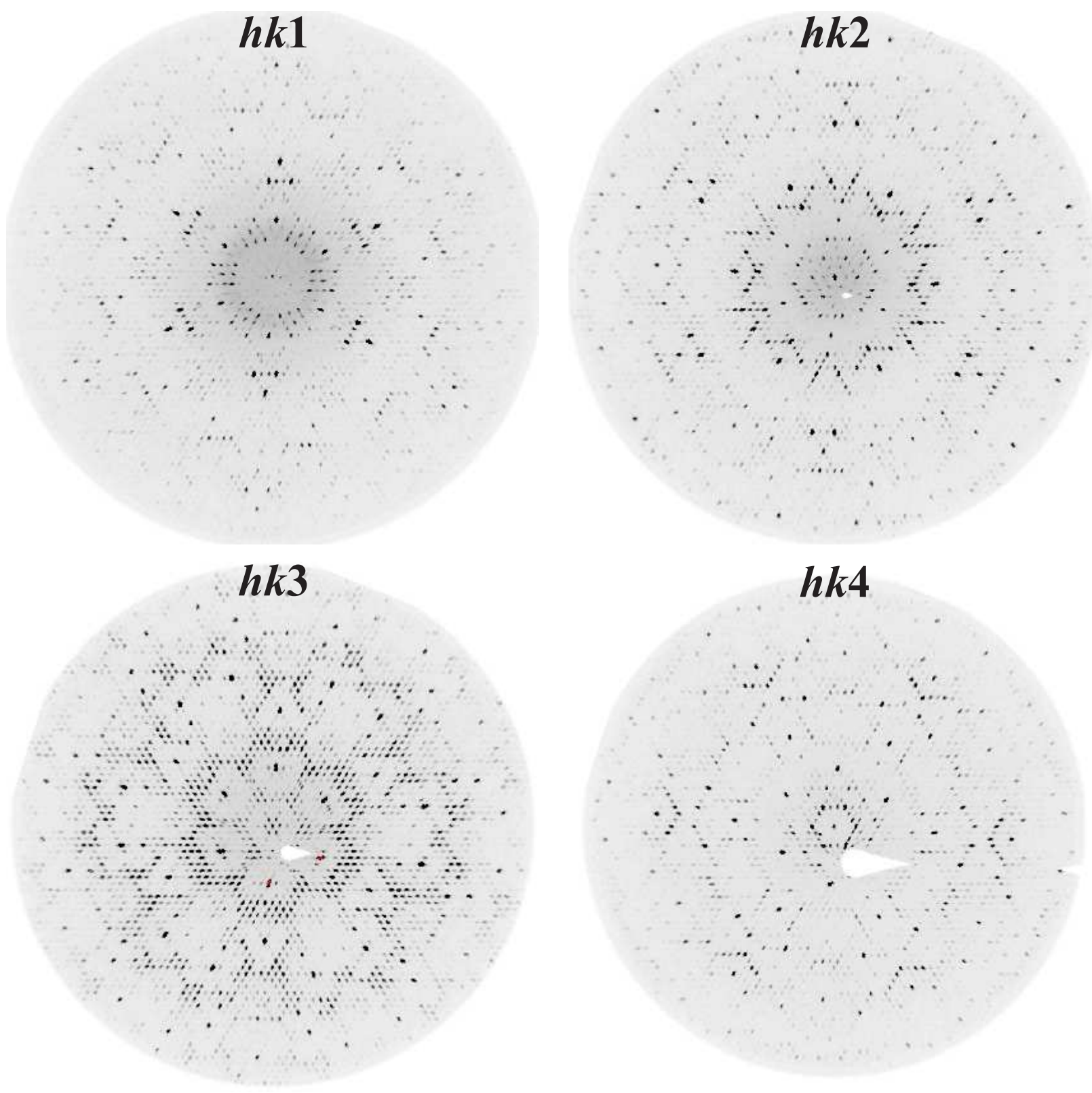

$h k 5$

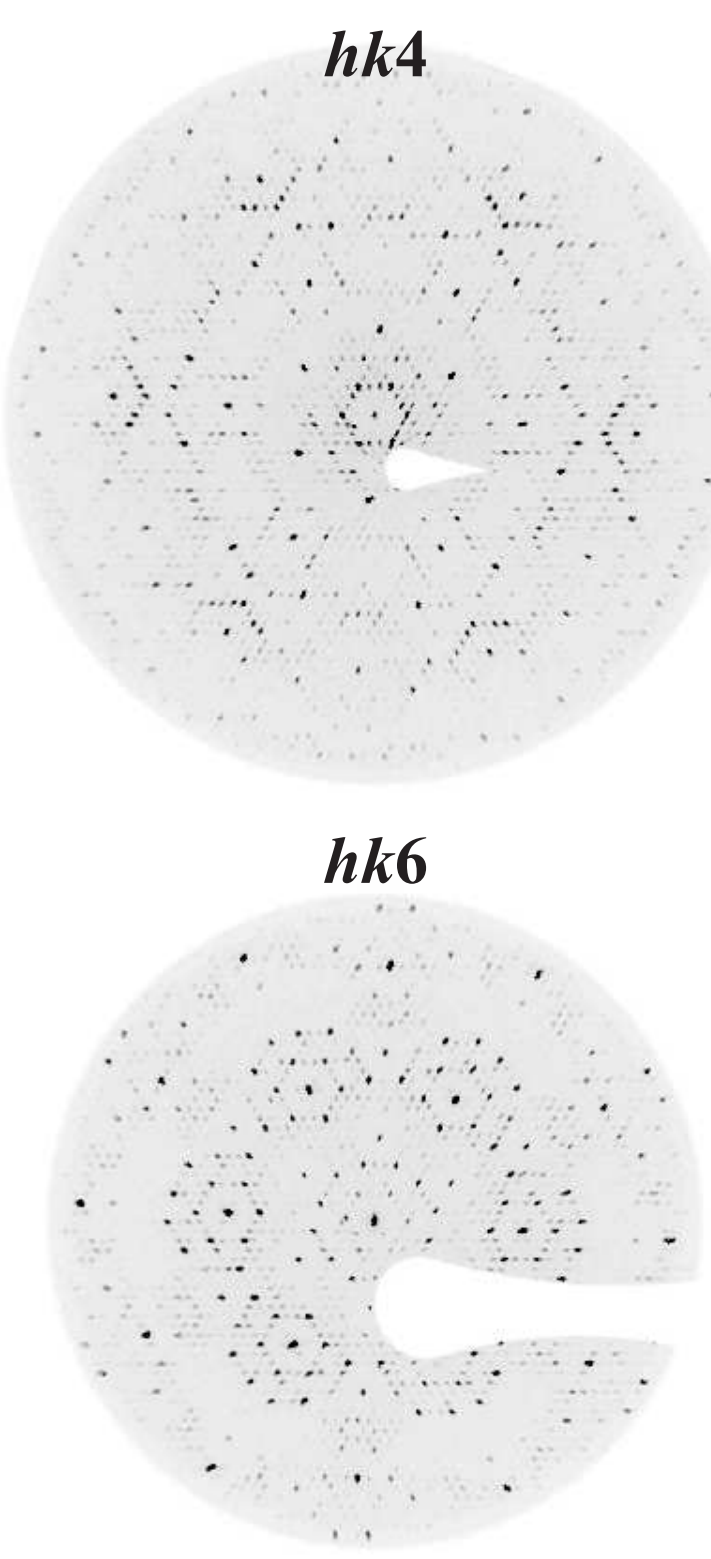


Figure 3: Reciprocal space sections of $\mathrm{Na}_{11} \mathrm{Hg}_{52}$ : $h 0 l$ to $h 4 l$.
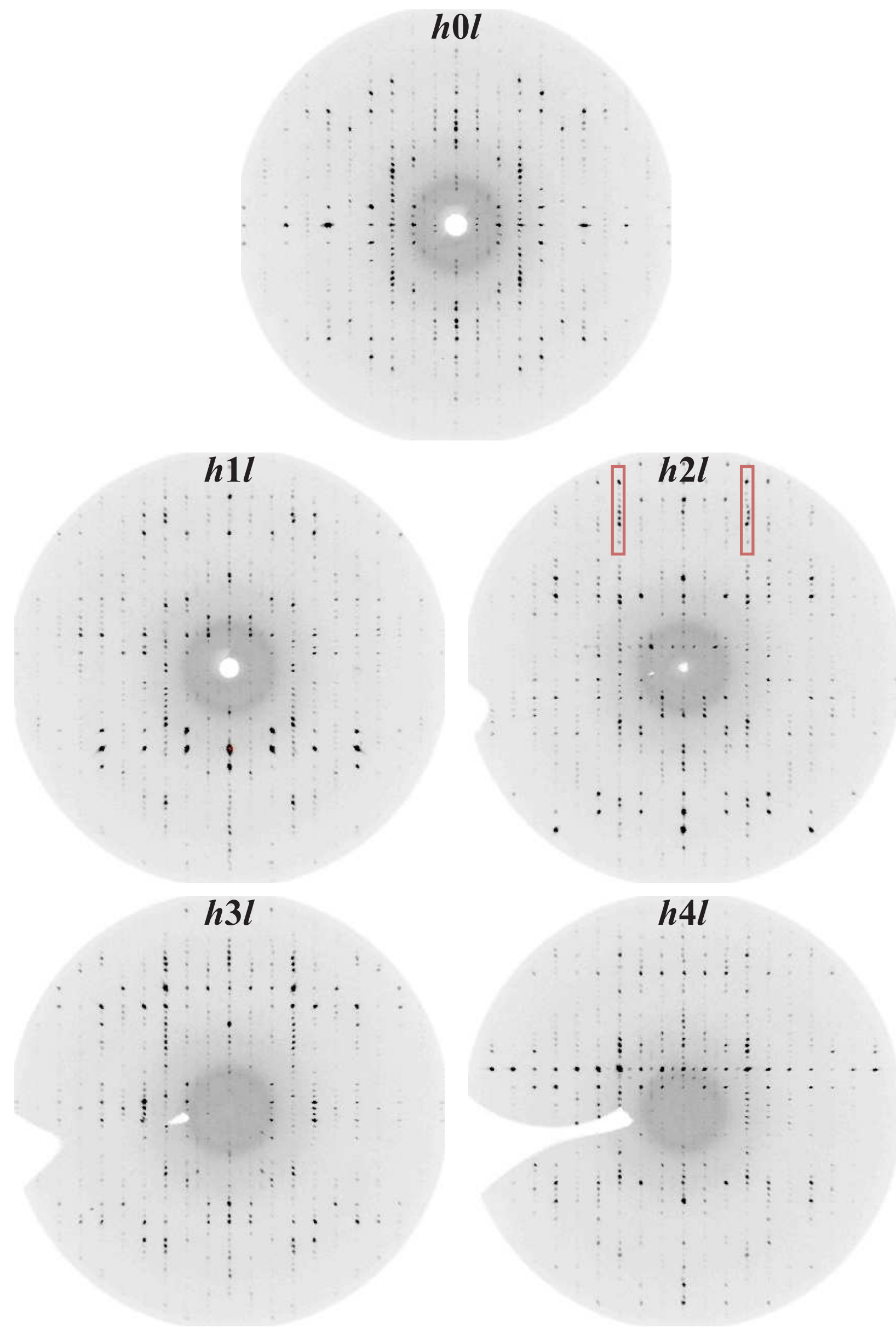
Figure 4: Reciprocal space sections of $\mathrm{Na}_{11} \mathrm{Hg}_{52}$ : $0 k l$ to $4 k l$.
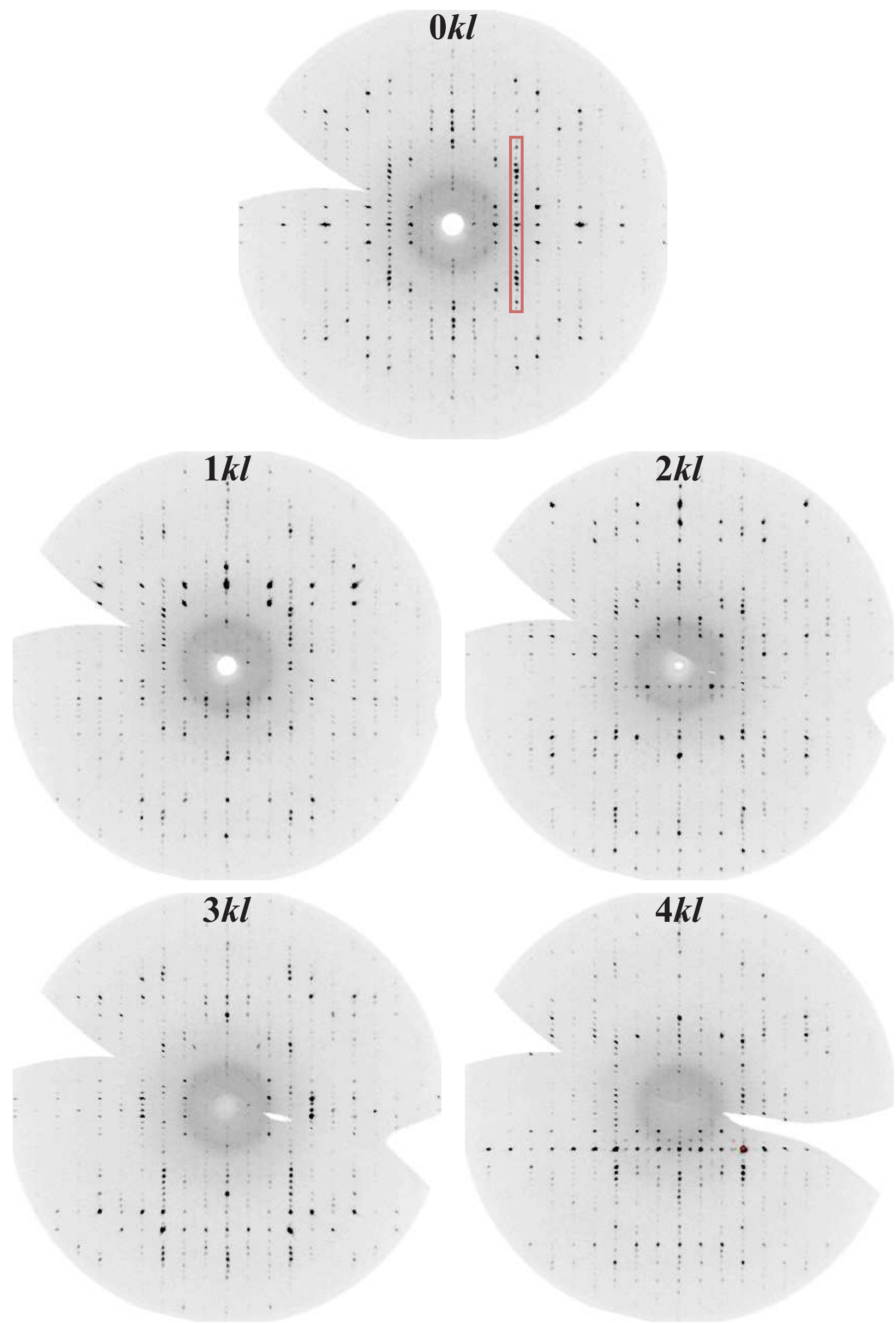


\subsection{Schematic diffraction patterns $h k 0$ of sublattices and partial structures used for the description of $\mathrm{Na}_{11} \mathrm{Hg}_{52}$}

In the following we show a collection of schematic diffraction patterns calculated for two-dimensional real space projections $x y 0$ of point patterns (shown within a unit square) and their corresponding reciprocal space sections $h k 0$. Intensities were calculated according to the formula:

$$
I_{h k}=S \cdot\left|\sum_{j=1}^{N} f_{j} \cdot \exp \left[2 \pi i\left(h x_{j}+k y_{j}\right)\right]\right|^{2},
$$

where $S$ is an arbitrary scaling factor (with values given for relative comparison) and $f_{j}$ was set to unity for all patterns, irrespective of the nature $\left(\mathrm{Na}\right.$ or $\mathrm{Hg}$ ) of the point scatterer. ${ }^{1}$ Reciprocal space sections are shown for $|h+k| \leqslant 40$.

The purpose of this presentation is twofold, being didactic on the one hand, by means of highlighting the diffracion signatures of a selection of idealized superlattices in general, and pragmatic on the other hand, in order to separate the individual scattering contributions of these sublattices with respect to the diffraction pattern of $\mathrm{Na}_{11} \mathrm{Hg}_{52}$. Although the actual reciprocal space intensity distribution is determined by the interplay of all partial structures, especially including all the actual deviations from the idealized description, the influence of the superstructure ordering remains strong enough to discern it in the diffraction pattern.

A note on the following (ab)use of language: Ideal designates a full sublattice (i.e. with all possible sites occupied), whereas actual denotes a defective one; yet even the defective sublattices are themselves idealizations to the actual crystal structure!

\footnotetext{
${ }^{1}$ Indeed, the diffraction from the appropriate union of partial structures, even if accounted for the strong difference in scattering contrast of $\mathrm{Na}$ and $\mathrm{Hg}$, is dominated by the spatial perfection of the idealized sublattice with $T(14,1)=183$. This means, that almost all of the actually observable detailed features of the diffraction pattern (in all their subtlety and splendour) result from deviations from the perfect order of the ideal sublattice.
} 
Figure 5: Ideal sublattice $T(14,1)=183, Y \equiv 14 X(\bmod 183)$ (top left); Ideal sublattice $T(9,5)=$ $169, Y \equiv 147 X(\bmod 169)$ (top right); Both aforementioned ideal sublattices combined (bottom left); Ideal sublattice $T(9,5)=61, Y \equiv 14 X(\bmod 61)$ (bottom right). $S=2 \times 10^{-4}$ always. Apart from the superstructure reflections reciprocal lattice points are highlighted and should not to be mistaken for intensities!
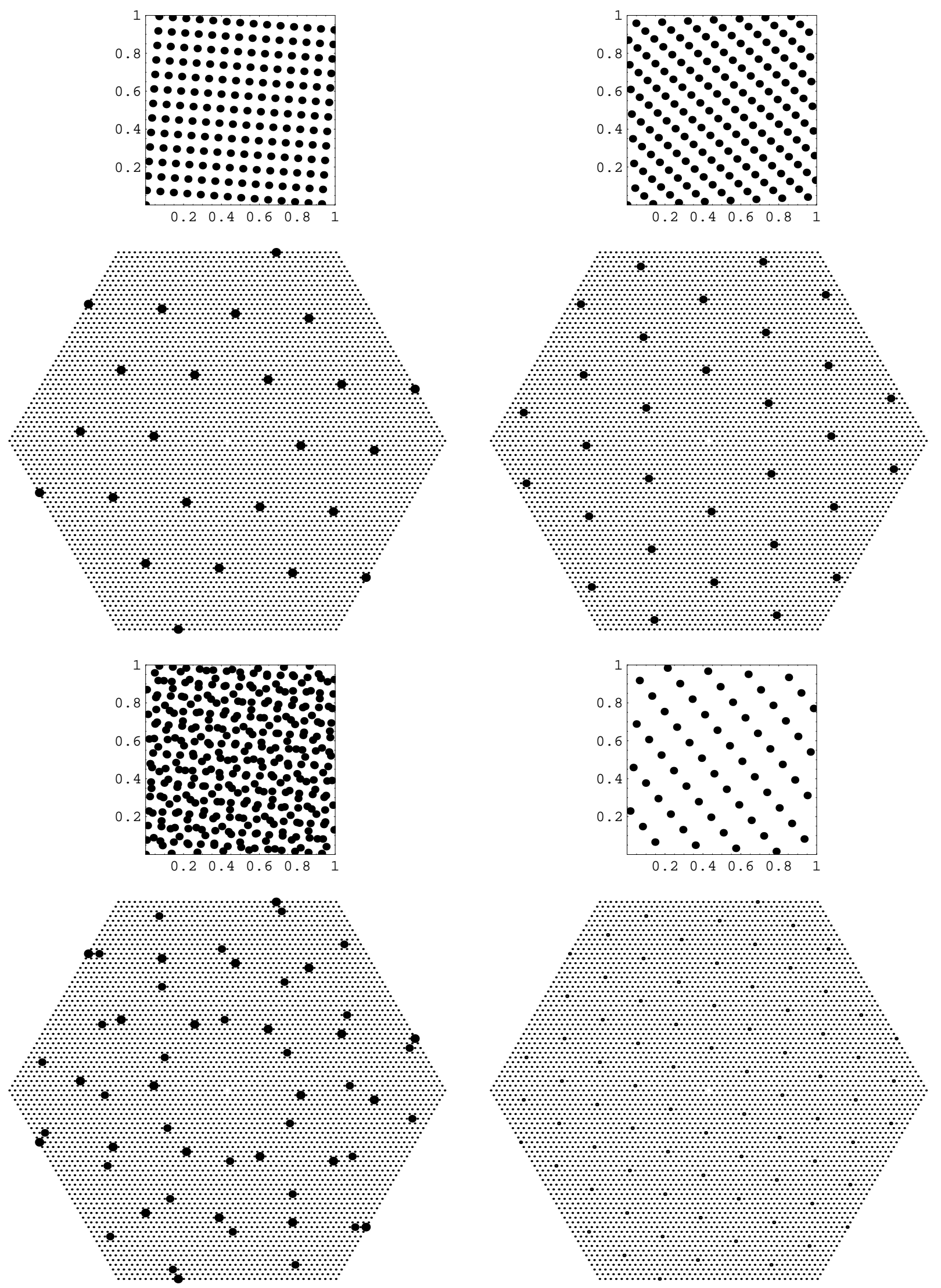


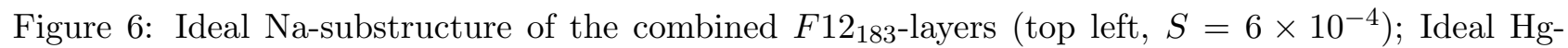
substructure of the combined $F 12_{183}$-layers (top right, $S=6 \times 10^{-4}$ ); Actual Na-substructure of the combined $F 12_{183}$-layers (bottom left, $S=6 \times 10^{-4}$ ); Actual Hg-substructure of the combined $F 12_{183}$-layers (bottom right, $S=6 \times 10^{-3}$ !).
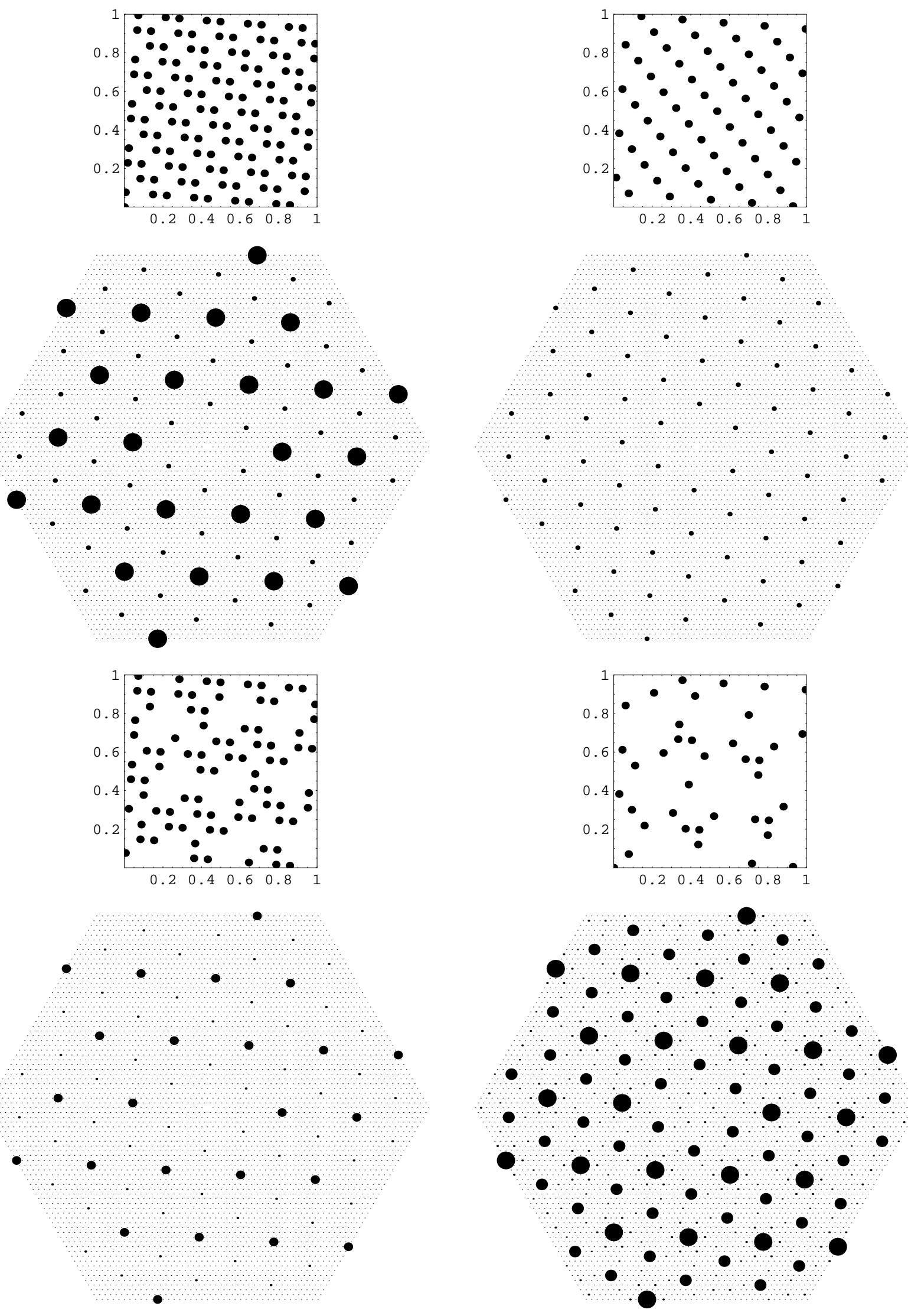
Figure 7: Actual Na-substructure of the $P$-layers (top left, $S=6 \times 10^{-2}$ ); Actual Hg-substructure of the $P$-layers (top right, $S=2 \times 10^{-4}$ ); Actual Na- and $\mathrm{Hg}$-substructure of the combined $F 12_{183}$-layers (bottom left, $S=2 \times 10^{-4}$ ); Actual Hg-substructure of the combined $F 12_{169}$-layers, with the second one shifted as described in the main body of the article (bottom right, $S=2 \times 10^{-3}$ ).

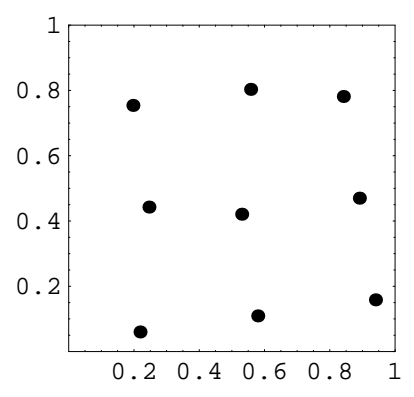

$\bullet \bullet \therefore \bullet \bullet \bullet \therefore \bullet \bullet \bullet \therefore \bullet$
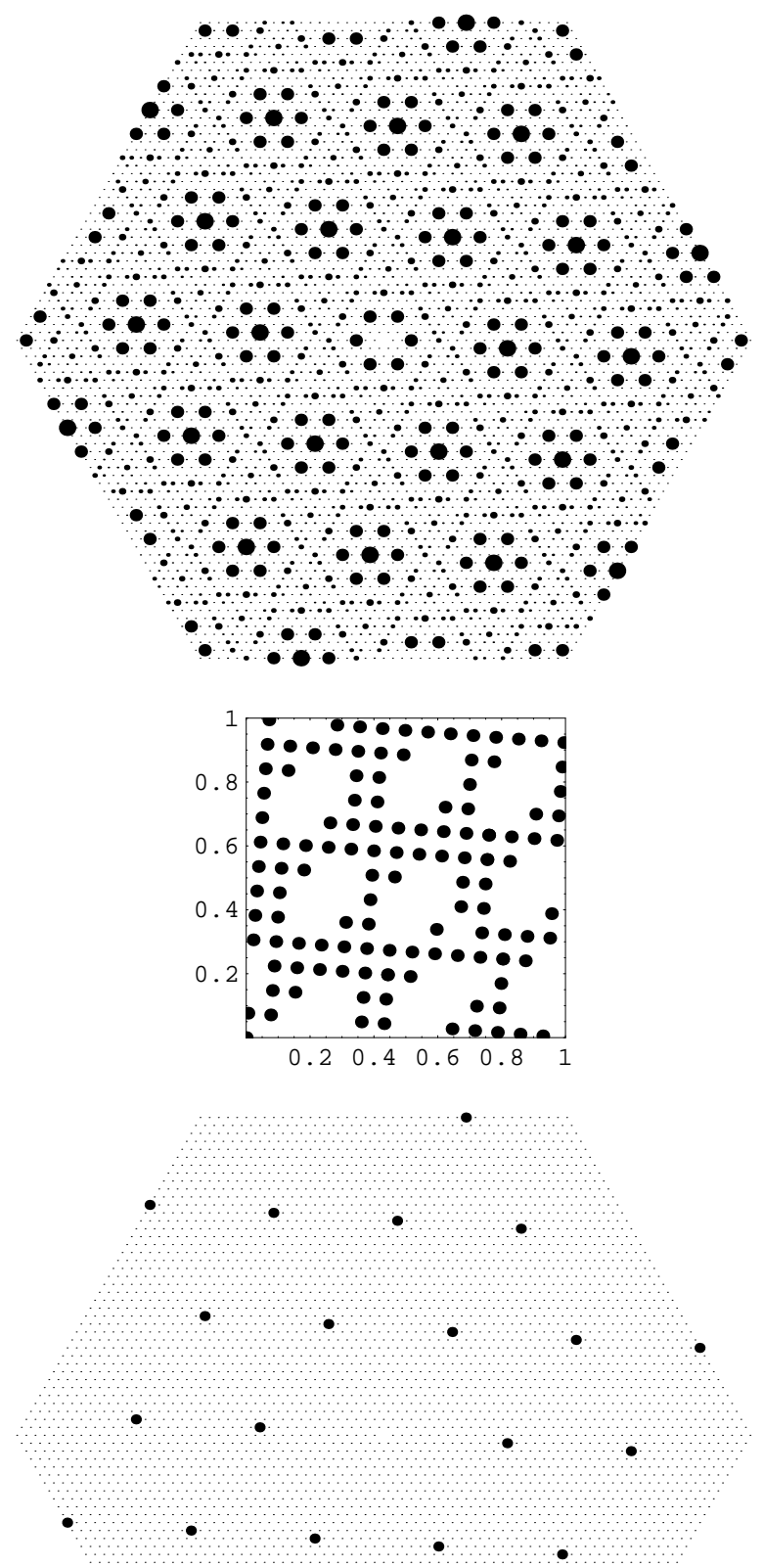

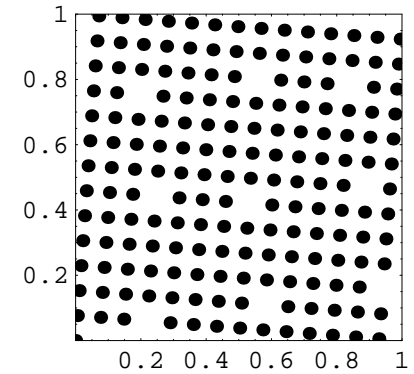

0
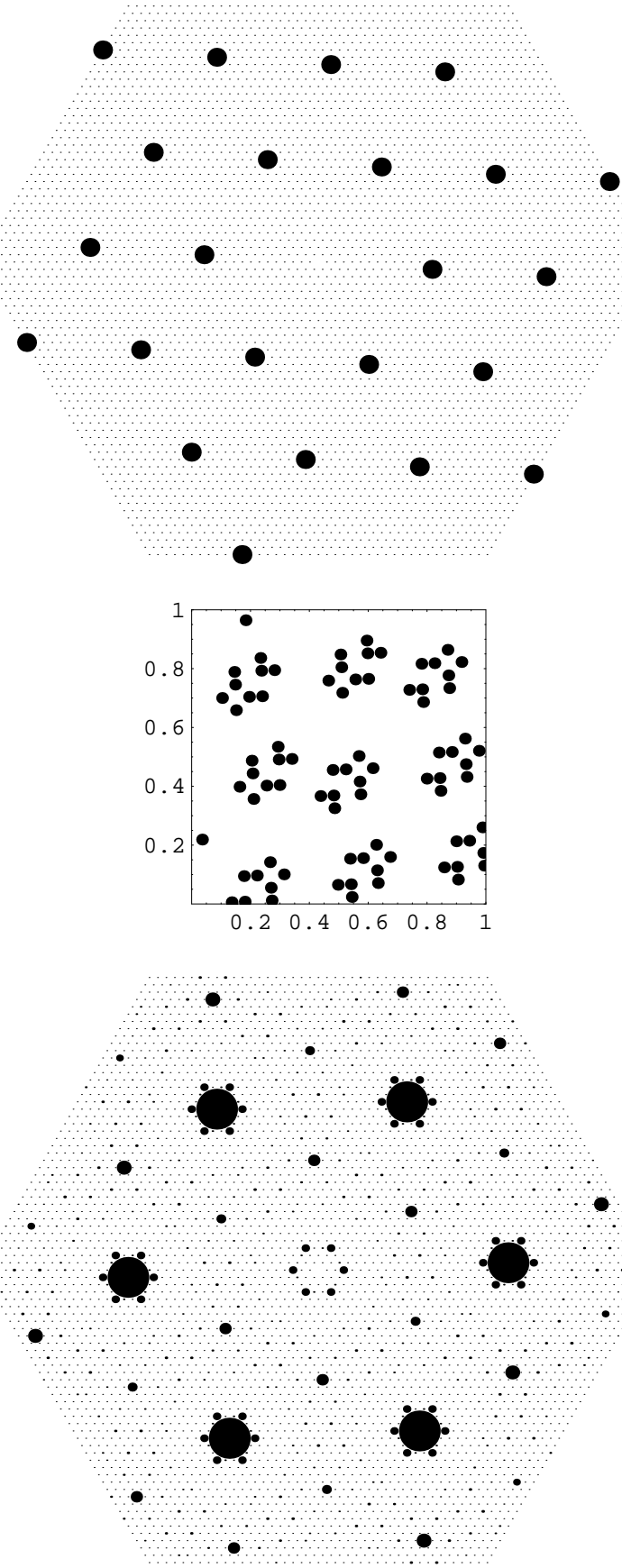


\section{Selection of enlarged figures}

Here, we show a few of the more complicated figures from the main article, especially concerned with the occupation of sublattice sites by sodium (lightgray) or mercury (darkgray) atoms.

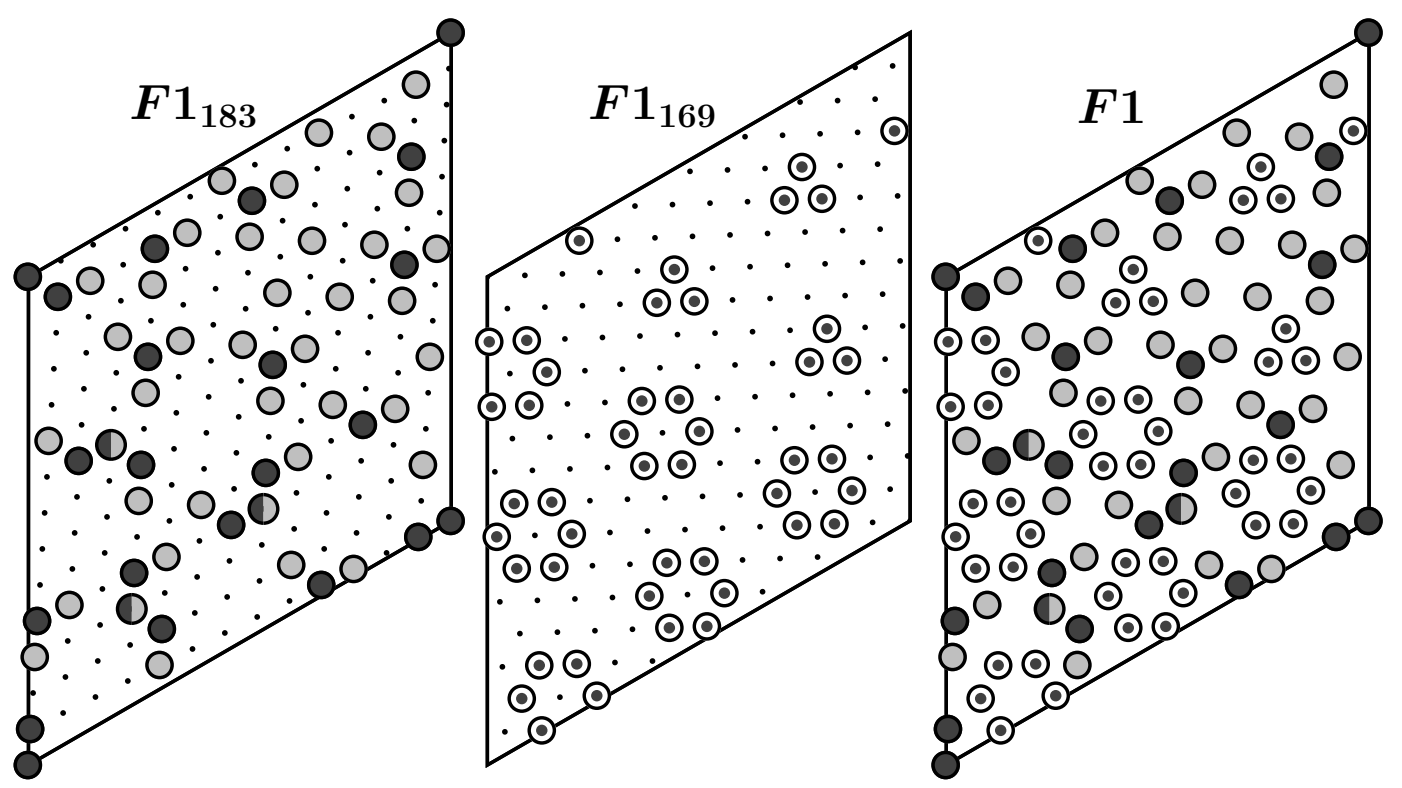

Figure 8: Idealized description of the $F$-layer by two sublattices of index $T(14,1)=183$ (left) and $T(15,7)=169$ (middle) and their combination (right).

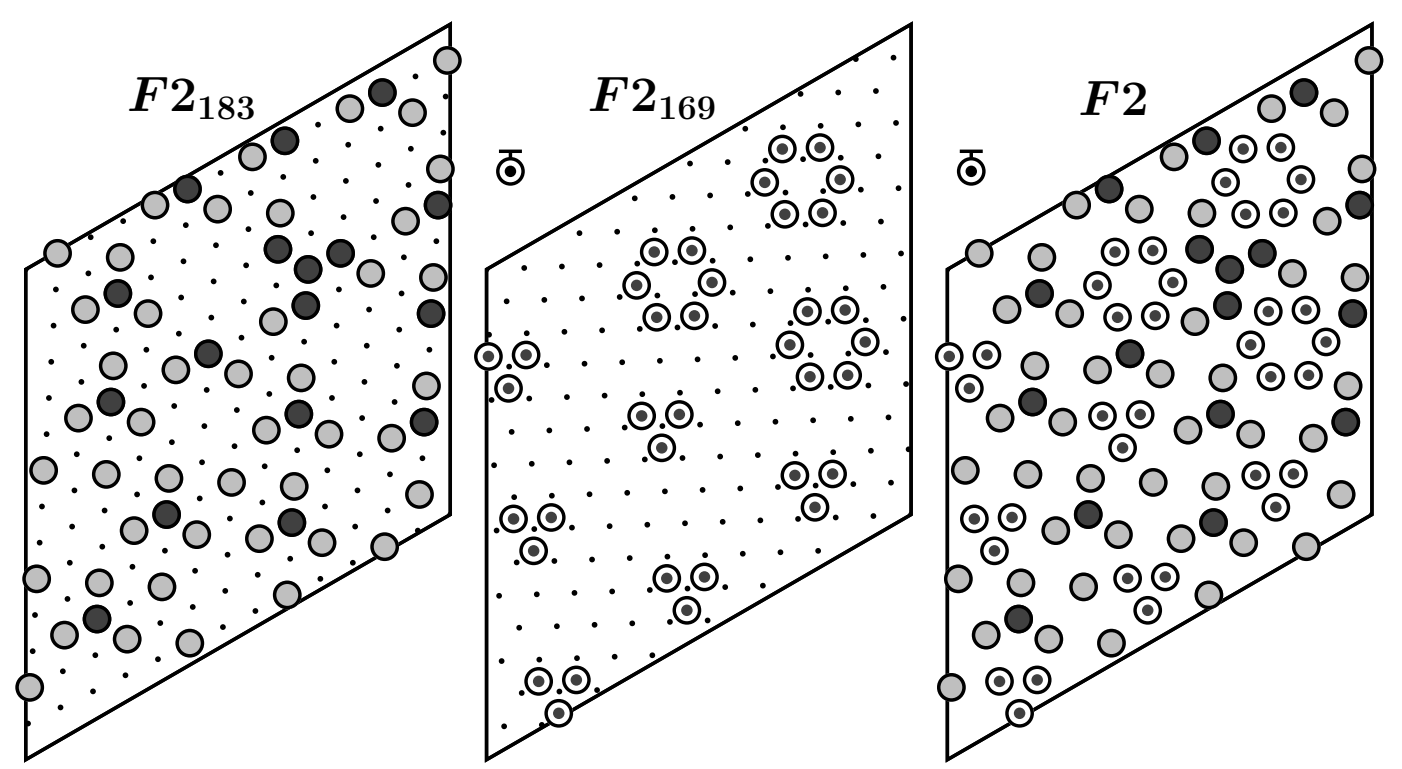

Figure 9: Idealized description of the $F$-layer by two sublattices of index $T(14,1)=183$ (left) and $T(15,7)=169$ (middle) and their combination (right). 

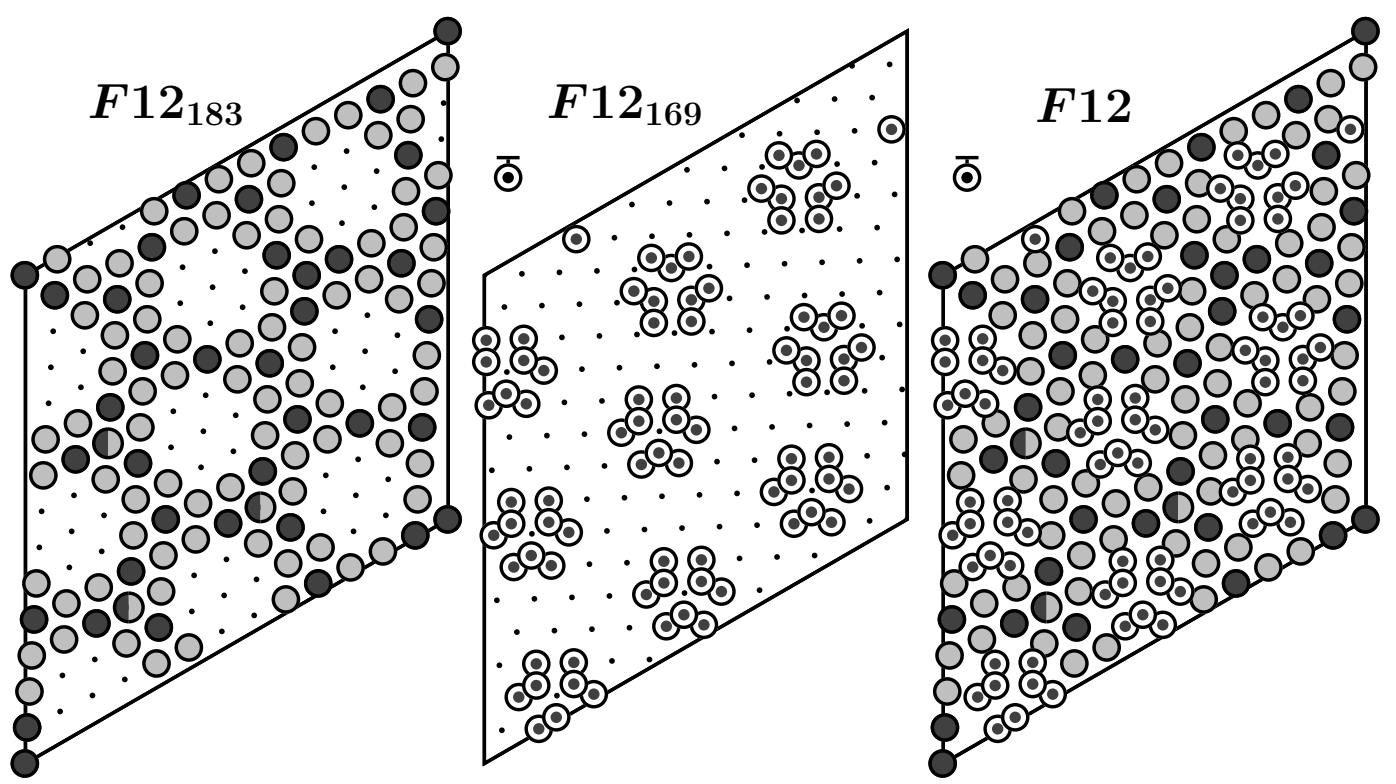

Figure 10: Combination of the idealized descriptions of the $F 1$ - and F2-layer by two sublattices of index $T(14,1)=183$ (left) and $T(15,7)=169$ (middle) and their meta-combination (right).

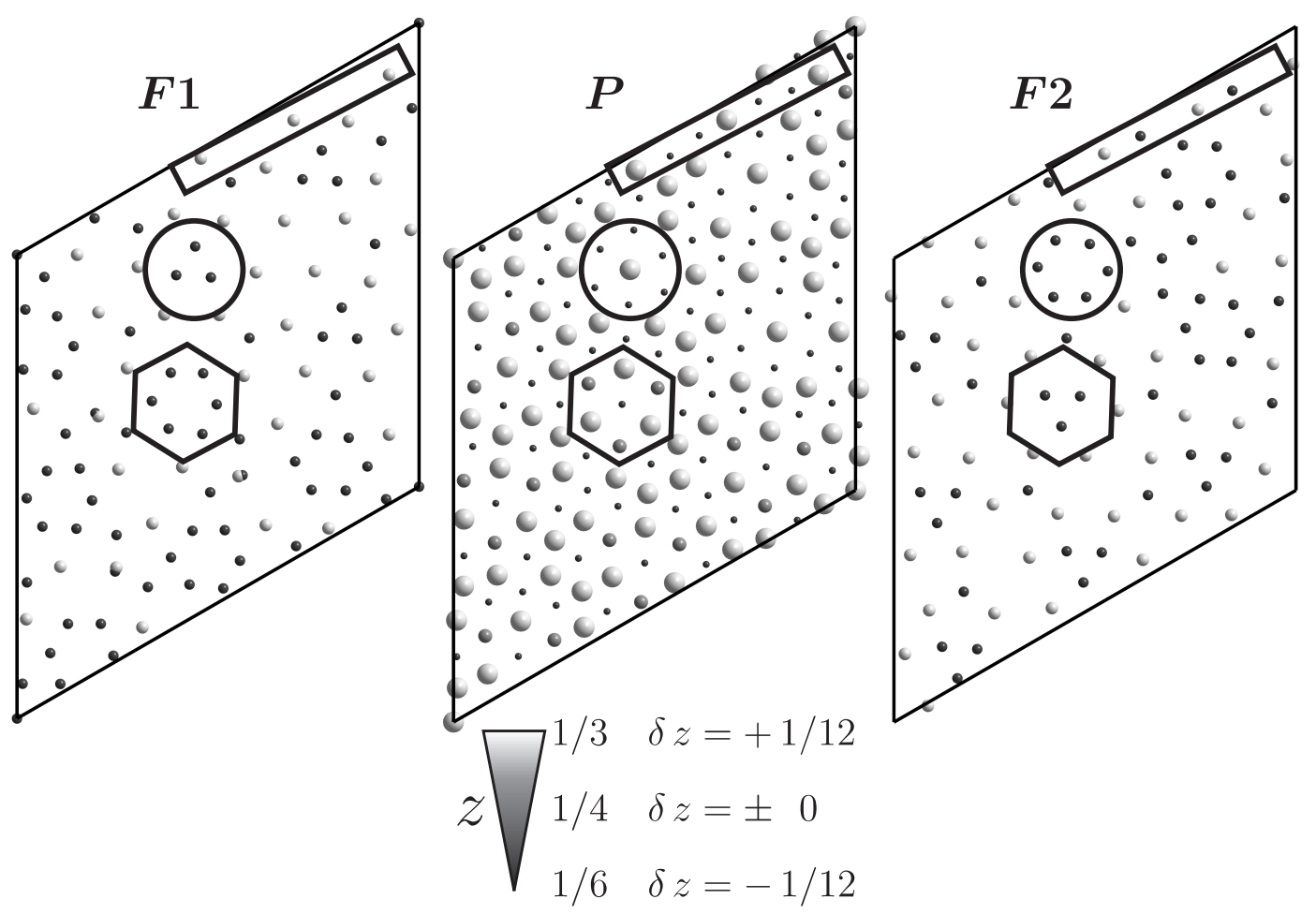

Figure 11: Structural correlation of the puckering of the $P$-layer (middle) with the occupancy of the adjacent $F 1 / F 2$-layers (left and right). Note, that the radii and gray values of the atoms within the $P$-layers do not represent the chemical decoration of the sublattice sites but instead denote the approximate height of the atoms in the $z$-direction, allowing for the depiction of atomic displacements $\delta z= \pm 1 / 12$ relative to the average height of the $P$-layer at $\langle z\rangle=1 / 4$. 


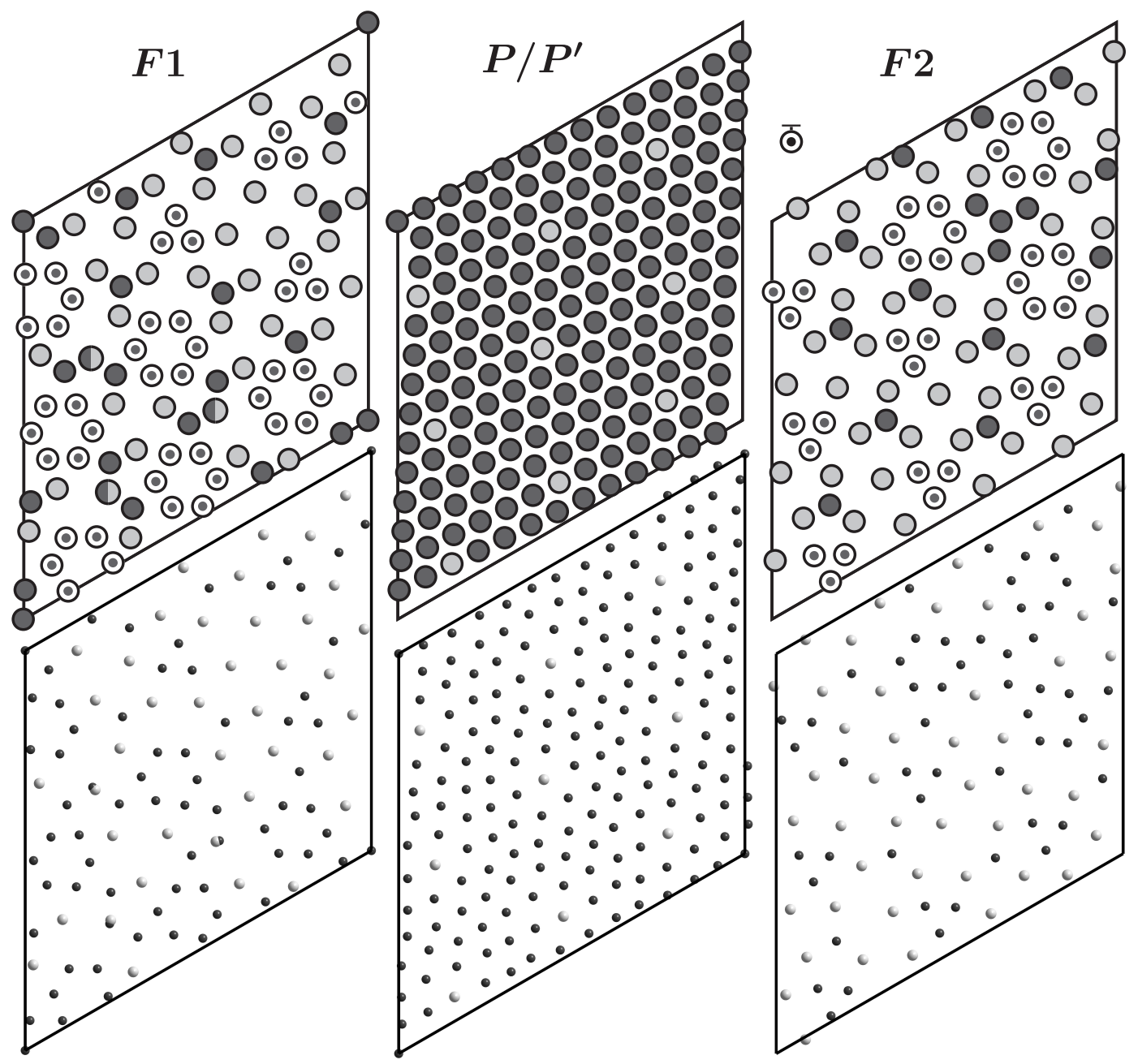

Figure 12: Comparison of the idealized layers (top) to the actual ones (bottom). Although slight deviations occur, the distribution of $\mathrm{Na}$ - and Hg-atoms is described quite well using sublattices and their MCG-encoding. 